\title{
Complex and extensive post-transcriptional regulation revealed by integrative proteomic and transcriptomic analysis of metabolite stress response in Clostridium acetobutylicum
}

Keerthi P. Venkataramanan ${ }^{1,2}$, Lie Min ${ }^{1,2}$, Shuyu Hou ${ }^{1,2}$, Shawn W. Jones ${ }^{1,2}$, Matthew T. Ralston ${ }^{1,3}$, Kelvin H. Lee , $^{1,2}$ and E. Terry Papoutsakis ${ }^{1,2^{*}}$

\begin{abstract}
Background: Clostridium acetobutylicum is a model organism for both clostridial biology and solvent production. The organism is exposed to its own toxic metabolites butyrate and butanol, which trigger an adaptive stress response. Integrative analysis of proteomic and RNAseq data may provide novel insights into post-transcriptional regulation.

Results: The identified iTRAQ-based quantitative stress proteome is made up of 616 proteins with a $15 \%$ genome coverage. The differentially expressed proteome correlated poorly with the corresponding differential RNAseq transcriptome. Up to $31 \%$ of the differentially expressed proteins under stress displayed patterns opposite to those of the transcriptome, thus suggesting significant post-transcriptional regulation. The differential proteome of the translation machinery suggests that cells employ a different subset of ribosomal proteins under stress. Several highly upregulated proteins but with low mRNA levels possessed mRNAs with long 5'UTRs and strong RBS scores, thus supporting the argument that regulatory elements on the long 5'UTRs control their translation. For example, the oxidative stress response rubrerythrin was upregulated only at the protein level up to 40-fold without significant mRNA changes. We also identified many leaderless transcripts, several displaying different transcriptional start sites, thus suggesting mRNA-trimming mechanisms under stress. Downregulation of Rho and partner proteins pointed to changes in transcriptional elongation and termination under stress.

Conclusions: The integrative proteomic-transcriptomic analysis demonstrated complex expression patterns of a large fraction of the proteome. Such patterns could not have been detected with one or the other omic analyses. Our analysis proposes the involvement of specific molecular mechanisms of post-transcriptional regulation to explain the observed complex stress response.
\end{abstract}

Keywords: Metabolite stress, iTRAQ proteome, Microarray, RNAseq, Integrative analysis, Post-transcriptional regulation

\footnotetext{
*Correspondence: epaps@udel.edu

15 Innovation Way, Delaware Biotechnology Institute, University of

Delaware, Newark, DE 19711, USA

${ }^{2} 150$ Academy Street, Department of Chemical and Biomolecular

Engineering, University of Delaware, Newark, DE 19711, USA

Full list of author information is available at the end of the article
} source, provide a link to the Creative Commons license, and indicate if changes were made. The Creative Commons Public Domain Dedication waiver (http://creativecommons.org/publicdomain/zero/1.0/) applies to the data made available in this article, unless otherwise stated. 


\section{Background}

Clostridium acetobutylicum is a model organism for the acetone, butanol, and ethanol (ABE) fermentation and for clostridial biology in general. C. acetobutylicum has the ability to ferment a very large range of carbon sources for the production of a wide array of products, including carboxylic acids (butyrate and acetate) and solvents $(\mathrm{ABE})[1,2]$. These products are toxic and affect cell growth and survival. A deeper understanding of the response and tolerance to metabolite and more generally chemical toxicity can lead to robust and rational design of strains suitable for industrial bioprocessing [1, 3-5]. More broadly, Clostridium organisms are predominantly soil bacteria that, in their natural milieu, are exposed to a large variety of chemicals, many of which are toxic to the cells. As a result, they have evolved to develop specific mechanisms to resist chemical toxicity $[6,7,4]$. Although response to chemical toxicity is not necessarily related to tolerance, several studies have demonstrated that components (genes/proteins, programs) of the wellpreserved heat shock protein (HSP or stress) response can be engaged to develop tolerant strains [4, 8-11]. We will show here that regulation of this core HSP response is considerably more complex that has been so far revealed by transcriptional studies. These are discussed next.

Several transcriptomic studies using DNA microarrays and, recently, RNAseq, have dissected the RNome dynamics of this organism. These studies have unveiled the transcriptional program associated with culture-phasespecific metabolism and physiological changes [12, 13]. Transcriptional analyses have also shed light into the dynamics of the organism's stress response to toxic fermentation products, notably butanol, butyrate, and acetate, in batch $[6,7,14-16]$ and continuous cultures $[17,18]$. Microarray data have been used to identify and characterize the stress-responsive gene network using phylogenetic RSAT footprinting analyses [16], while the RNAseq studies have been used to identify stress-responsive non-coding small RNAs (sRNAs) that might be part of the regulatory network of the stress response [15].

In view of the complex post-transcriptional events, such as differential mRNA and protein stability, differential regulation of the translation process, and the involvement of regulatory sRNAs, integrative analysis of transcriptomic and proteomic data could provide many novel insights not possible using only one type of omic data [19].

Here, we present the analysis of a large set of proteomic data aiming to examine at the proteome level the response of $C$. acetobutylicum to butanol and butyrate stress and a comparative analysis of these proteomic data against two sets of transcriptomic data, one based on microarray analysis [16] and the second on RNAseq
[15]. These extensive omic data were collected from the same master cultures. Their analysis aims to provide a more comprehensive understanding of the metabolite stress response with emphasis on identifying new regulatory mechanisms not accessible through either transcriptomic or proteomic analyses alone.

\section{Results}

The metabolite stress proteome of $C$. acetobutylicum A large and deep set of proteomic data to characterize the dynamic cellular response to butanol and butyrate stress

C. acetobutylicum cultures were grown anaerobically in 4. L bioreactors at $37{ }^{\circ} \mathrm{C}$ on defined CGM with $40 \mathrm{~g} / \mathrm{L}$ glucose. As in the corresponding microarray [16] and RNAseq [15] studies, cultures were stressed with three levels of butyrate ( $0 \mathrm{mM}$ - control; $30 \mathrm{mM}$ - low; $40 \mathrm{mM}$ medium; and $50 \mathrm{mM}$ - high) and three levels of butanol (0 mM - control; $30 \mathrm{mM}$ - low; $60 \mathrm{mM}$ - medium; and $90 \mathrm{mM}$ - high) stress at a cell density $\left(\mathrm{A}_{600}\right)$ of 1.0. The effect of butanol stress was dose dependent and had a severe impact on cell growth and glucose utilization in comparison to the non-stressed control cultures (Additional file 1: Figure S1) On the other hand, although butyrate stress affected substrate utilization, its impact on cell growth was not severe, as the growth of the butyratestressed cultures was similar to the non-stressed control cultures. Clostridial metabolism includes acid reassimilation leading to solvent formation. It appears that uptake of the exogenous butyrate minimizes the impact of this carboxylic acid on cell growth $[12,15,16]$.

The stress proteome was identified using iTRAQ (4-plex) samples from 15,45 , and 75 min post stress and a reference pool that was created by pooling equal amounts of proteins from all samples for each metabolite stress (see Materials and methods). A total of 440 and 589 proteins (Fig. 1, panels a, b, and c) were identified under butanol and butyrate stress, respectively. Four hundred thirteen proteins were detected under both butanol and butyrate stress (Fig. 1). Stressed samples were compared against the non-stress sample to identify proteins that were expressed only during stress and proteins that were expressed only during non-stress control condition. Comparing the proteome of butanol-stressed sample with its corresponding non-stressed control, 90 proteins were exclusively detected only under butanol stress conditions with no expression under non-stress control, while 44 proteins were exclusively detected only under the corresponding control, non-stress conditions with no expression under butanol stress (Figs. 1 and 2 and Additional file 1: Figure S2). Similarly, between butyrate-stressed and non-stress control samples, 120 and 67 proteins were exclusively detected only under butyrate-stress versus the corresponding control, non-stress conditions, respectively (Figs. 1 and 3 Additional file 1: Figure S2). Proteins 


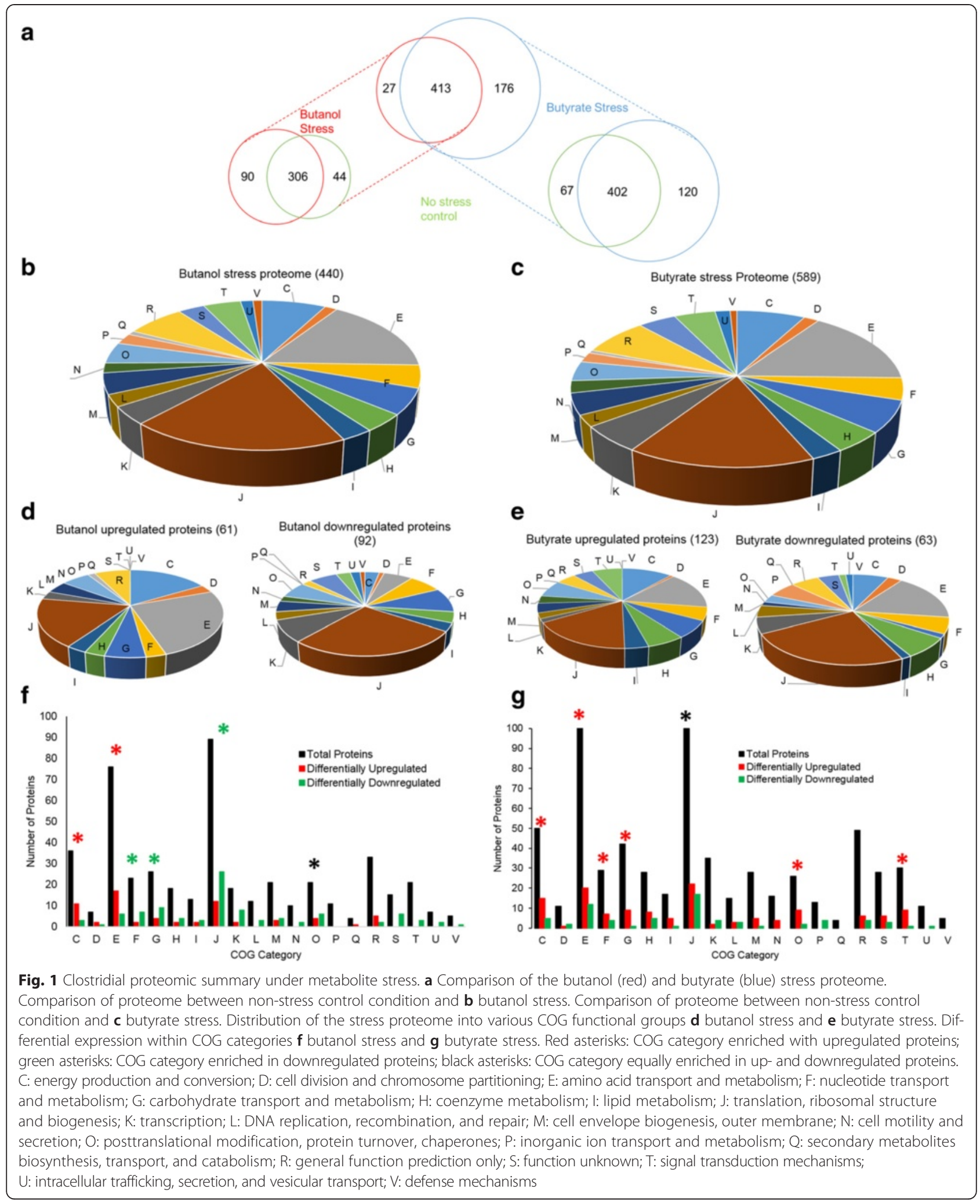

expressed only under either stress or control conditions likely have an important role in these metabolite stress responses.
These data give a first glimpse of what will be reinforced below by analysis, namely that the butyrate stress proteome is considerably larger than the proteome of the 


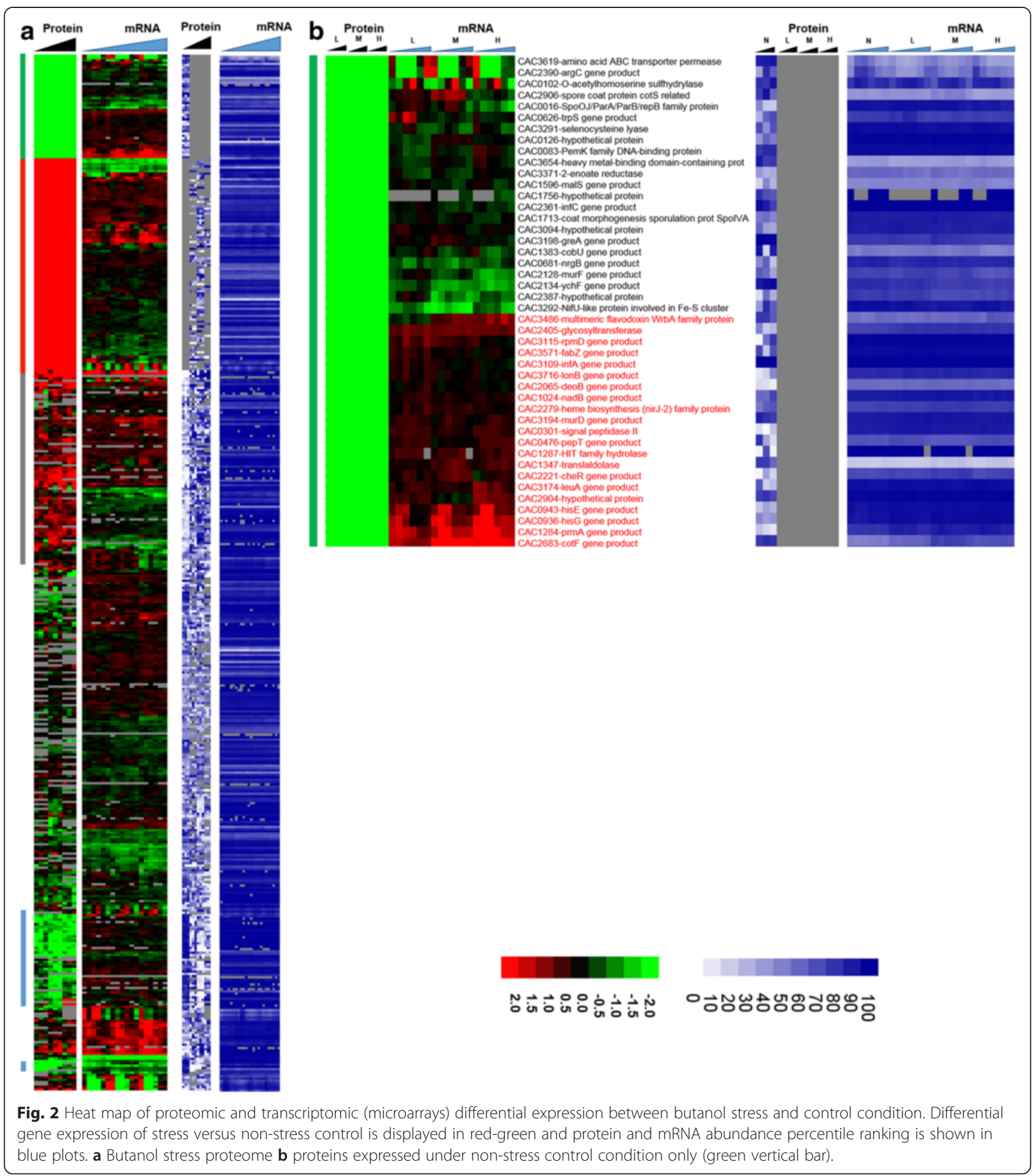

butanol stress. This is based on the logical assumption that there is no bias from sample to sample in protein detection by the method employed here.

\section{Differential expression of proteins under metabolite stress}

Although, as already discussed, several studies have examined the metabolite stress response at the transcriptional level, relatively little is known about the stress proteome. As we shall show here, the detectable proteome displays distinct differences from the transcriptome, thus suggesting a more complex regulation that was anticipated from the transcriptomic studies. Analysis of differential protein expression was carried out by pairwise comparison of each time point between each stress condition and the 


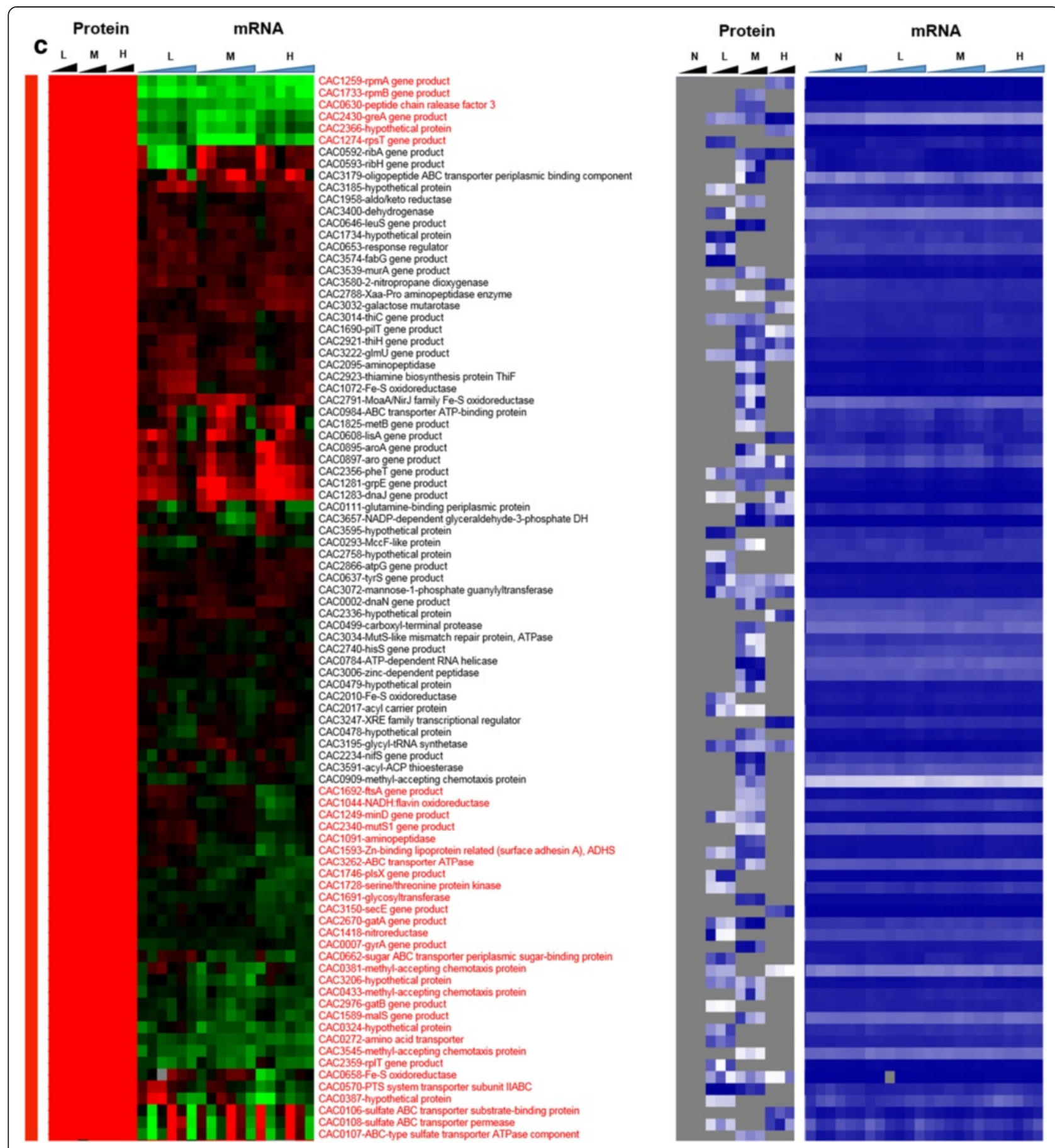

Fig. 2 (continued) c proteins expressed only under stress (red vertical bar)

corresponding non-stress condition using MeV's SAM analysis (see Materials and methods); with the additional, typical requirement of a fold change $\geq 2.0,306$ proteins (Fig. 1) that were detected under both control and a given stress condition (low, medium or high) were considered for differential expression analysis under butanol stress. Among those, 243, 280, and 222 proteins were used for the analysis under low, medium, and high butanol stress, respectively, out of which 48,76 , and 75 proteins, respectively, were found to be significantly (fold change $\geq 2.0$ ) differentially expressed. Similarly, under butyrate stress, 337, 344, and 357 proteins (a total of 402 proteins, Fig. 1) were used to analyze differential expression at low, medium, and high butyrate stress, out of which 55,64 , and 58 proteins, respectively, were found to be differentially expressed. 


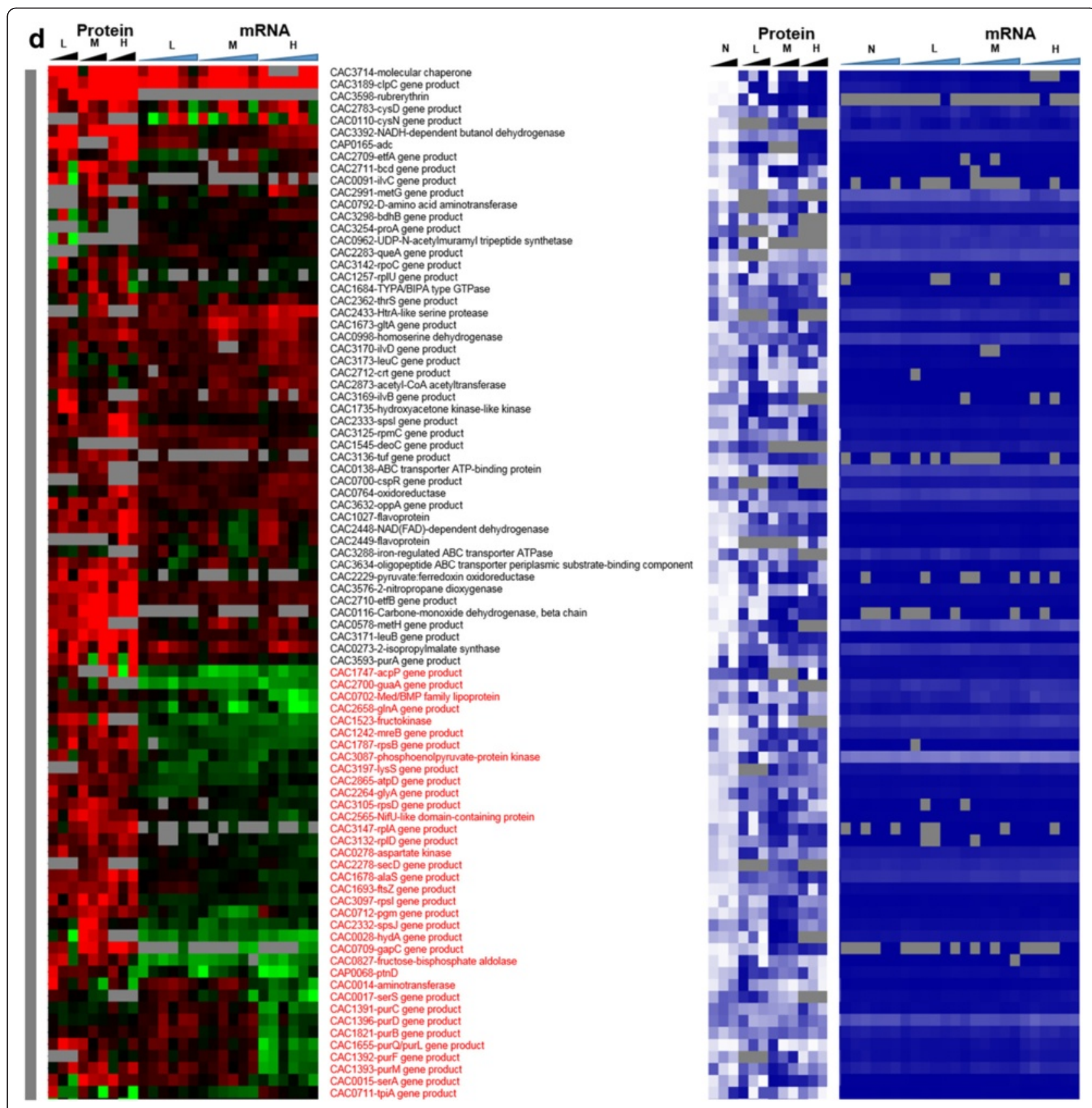

Fig. 2 (continued) d significantly upregulated proteins under stress (grey vertical bar)

Under both stress conditions, there was a set of proteins that were only under the control non-stress condition. These consisted of 44 and 67 proteins under butanol and butyrate stress, respectively (Table 1, Fig. 1, and Additional file 1: Figure S2). Similarly, several proteins were found to be expressed only under the stress condition and not under the control condition. These consisted of 90 and 120 proteins under butanol and butyrate conditions (Table 1, Fig. 1, and Additional file 1: Figure S2), respectively. These proteins/genes were classified as differentially expressed. Because it is not possible to calculate the fold difference for proteins expressed only under one (stress or control) condition, these two sets were assigned the maximum value observed for differentially (6.0) up- or downregulated $(-5.0)$ proteins (Figs. 4 and 5). Some of the key proteins that were detected only under stress (which means that their expression levels under no stress were below detection limits) are summarized in Tables 2 and 3. These proteins belong to functional groups relevant to the stress response and the physiology of the cells, notably, to 


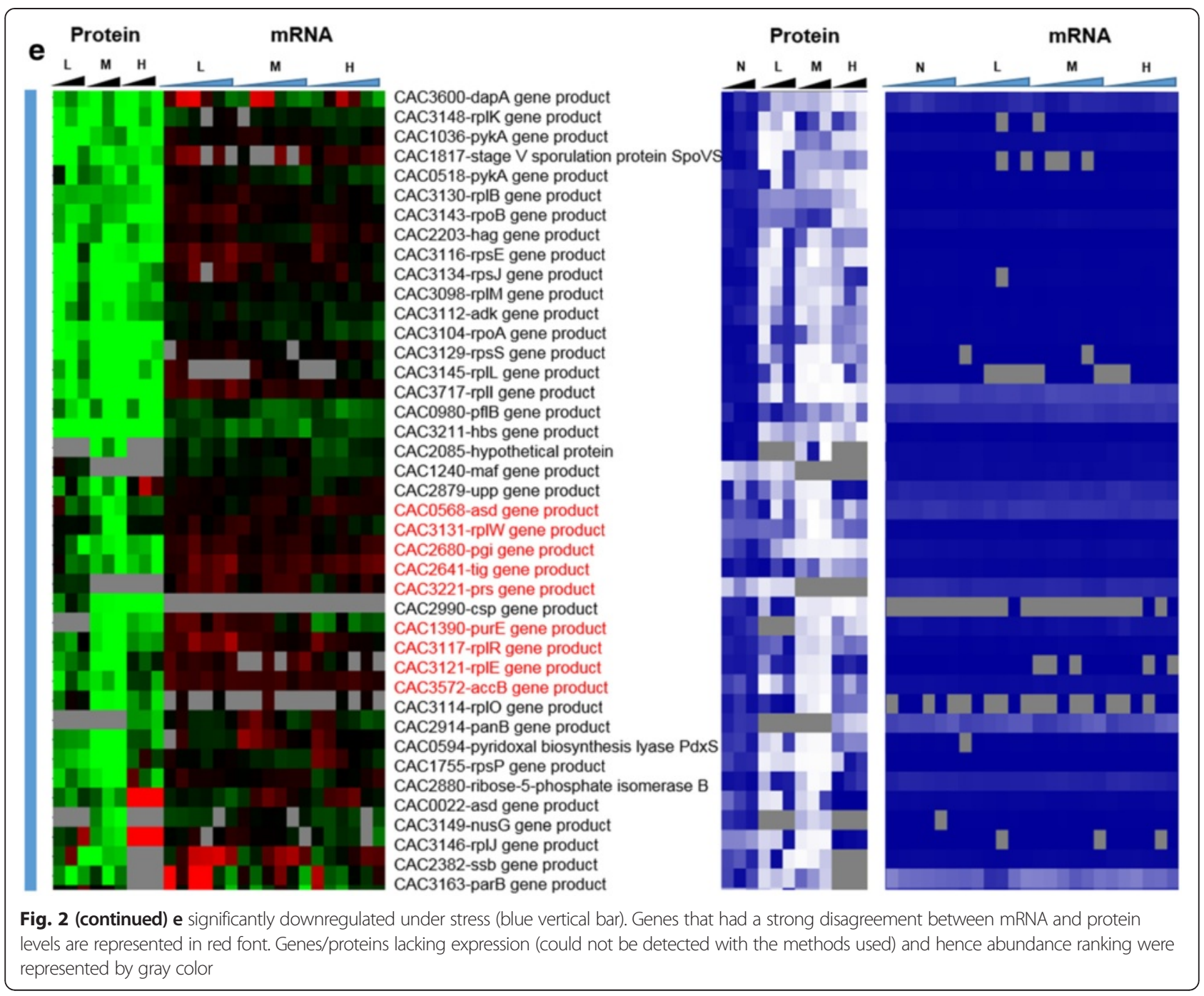

heat shock proteins (HSPs), UV stress response proteins, transcriptional regulators, response regulators involved in signal transduction, and chemotaxis proteins. Of note is that HSP proteins YacH and YacI in the $c l p C$ operon [16] were detected as expressed only under high butyrate stress but not under butanol stress.

Under butanol stress, the functional groups containing proteins from the translational machinery ( $\mathrm{J})$, nucleic acid metabolism (F), and carbohydrate metabolism (G) were enriched in differentially downregulated proteins. Groups of energy metabolism (C) and amino acid metabolism (E) were enriched in differentially upregulated proteins (Fig. 1). Under butyrate stress, compared to butanol stress, more COG groups were enriched in upregulated proteins: COG groups $C$, E (amino acid metabolism and transport), F, G, O (post-translational modification, protein turnover, and chaperone functions), and $\mathrm{T}$ (signal transduction) were predominantly upregulated.

Downregulation of proteins of the translational machinery (COG category J) under butanol stress is consistent with the observed growth inhibition. In contrast, there was substantially less downregulation of proteins in COG category J under butyrate stress, whereby there was no growth inhibition observed. Other differences between butanol and butyrate stress were in the expression of proteins involved in energy production (COG category G) and nucleic acid metabolism (COG category F), which may explain the uninhibited growth of cells under butyrate stress, compared to growth inhibition under butanol stress. Additionally, the upregulation of these pathways under butyrate stress supports the reassimilation of butyrate as observed in transcriptomic studies $[16,12,6,7,17,18,14]$. 


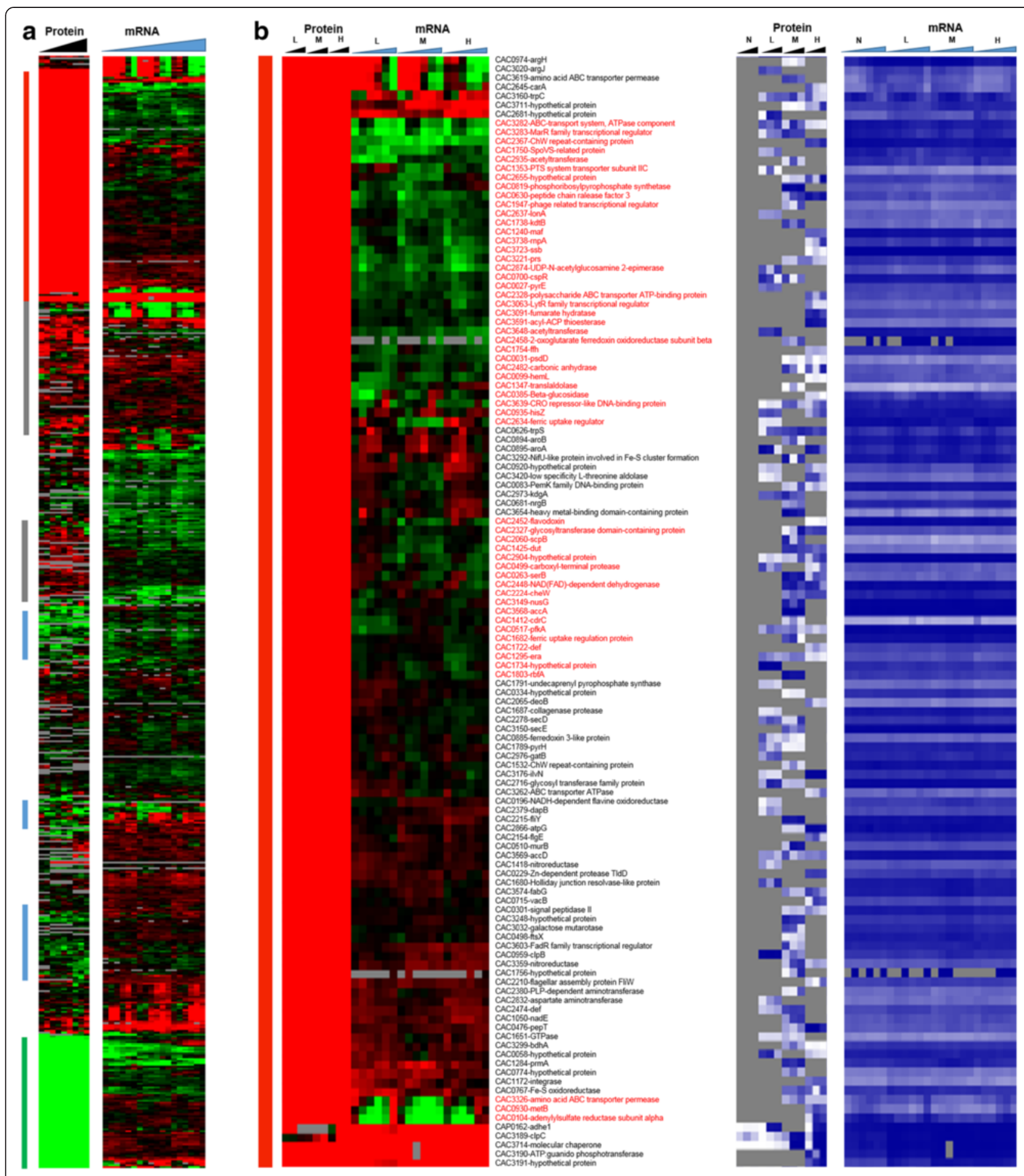

Fig. 3 Heat map of proteomic and transcriptomic (microarrays) differential expression between butyrate stress and control condition. Differential gene expression of stress versus non-stress control is displayed in red-green and protein and mRNA abundance percentile ranking is shown in blue plots. a Butyrate stress proteome b proteins expressed under stress (red vertical bar). Expression scales are with panel (e) 

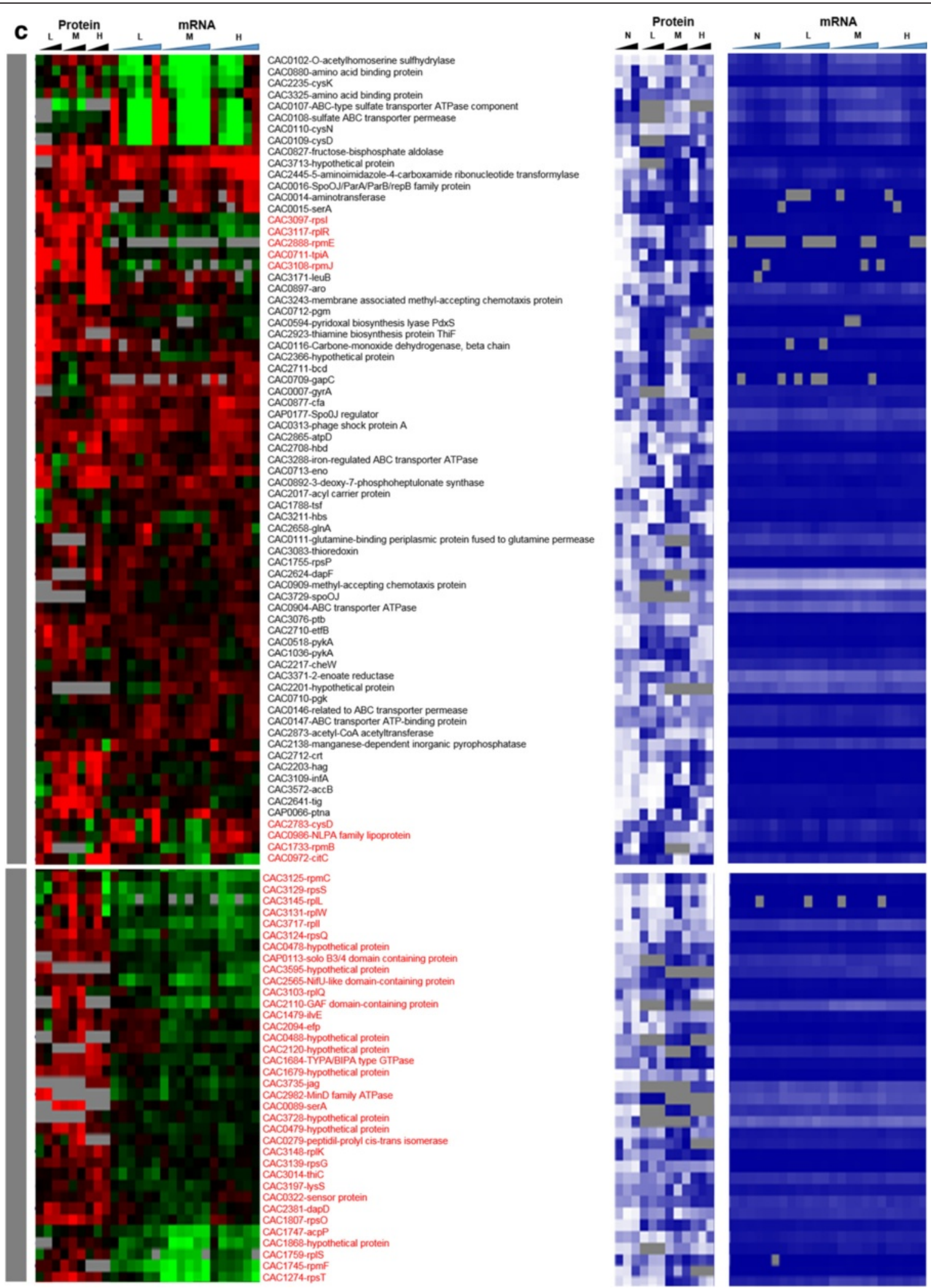

Fig. 3 (continued) c significantly upregulated proteins under stress (grey vertical bar). Expression scales are with panel (e) 


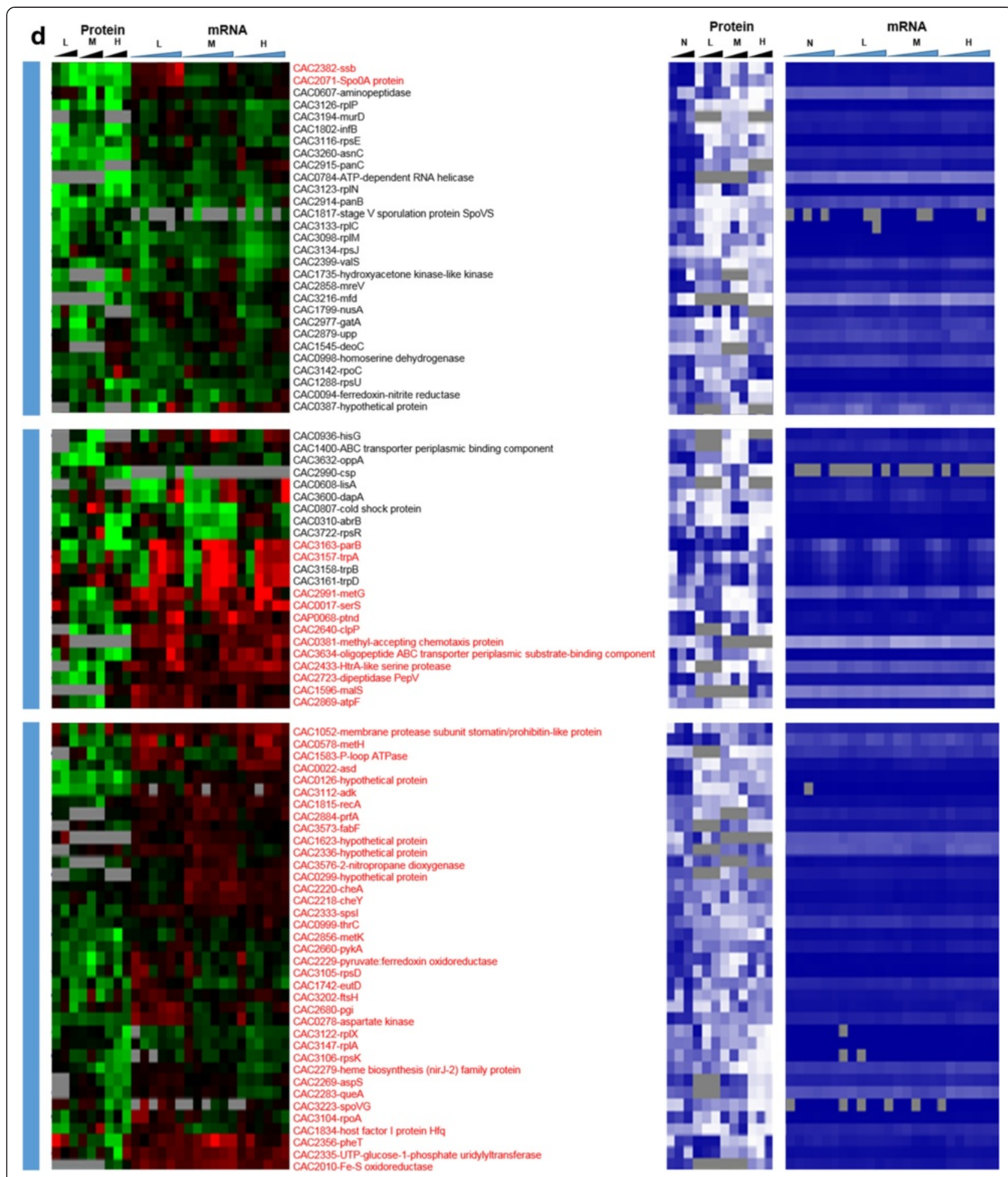

Fig. 3 (continued) d significantly downregulated under stress (blue vertical bar). Expression scales are with panel (e) 


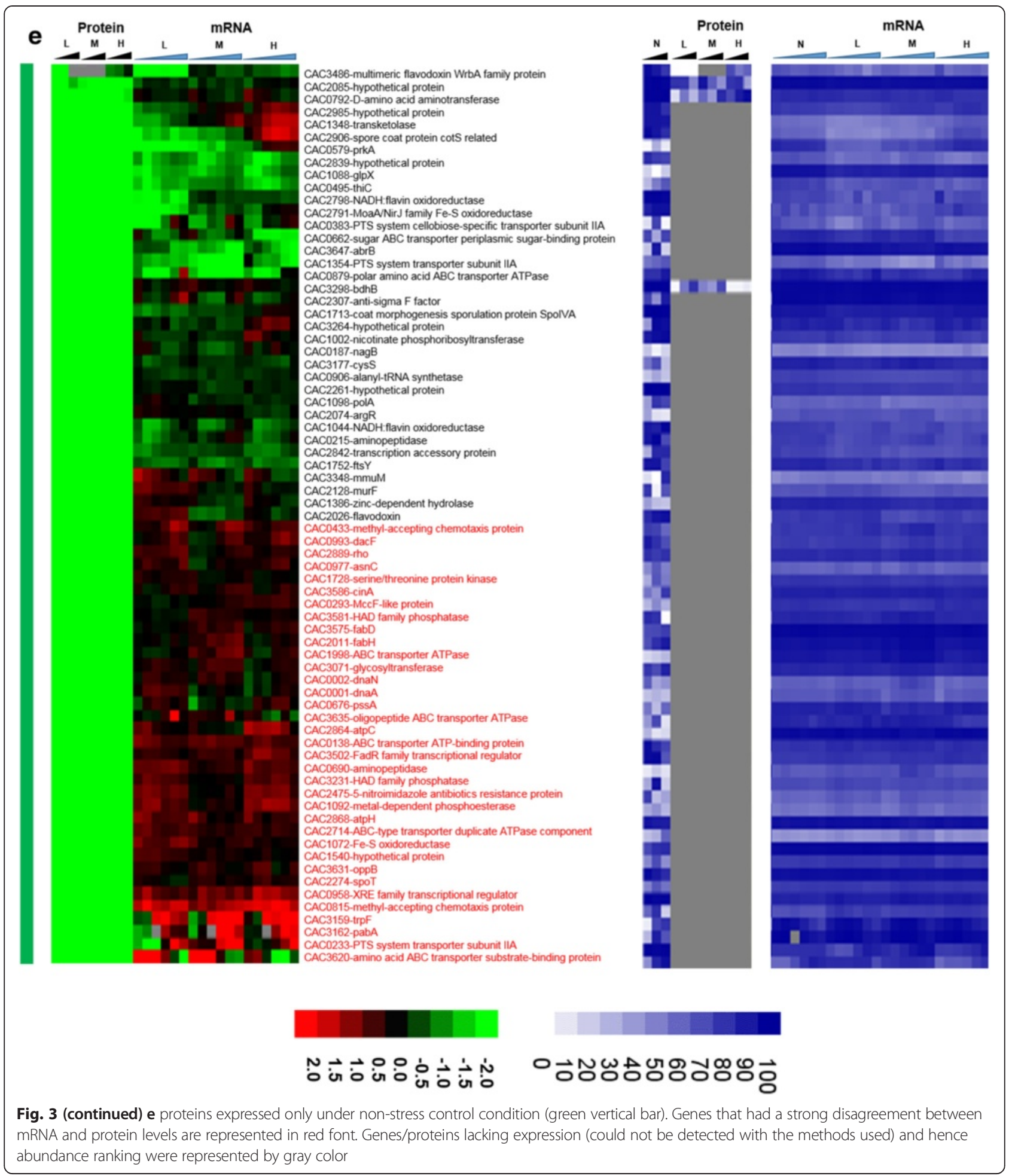

The global proteome versus the transcriptome under metabolite stress

\section{Global comparison demonstrates more complexity that cannot be anticipated from the transcriptomic data}

To probe whether proteomic and transcriptomic data agree in trends and patterns, we analyzed the data in several ways. First, we compared differential expression based on the proteomic data with the differential expression of the corresponding mRNAs using transcriptomic data from both RNAseq [15] and microarray analyses [16] (Fig. 2). The heat maps of this comparison make it possible to quickly identify genes/proteins, which are in 
Table 1 Differential expression analysis of proteomic data

\begin{tabular}{|c|c|c|c|c|c|c|}
\hline \multicolumn{7}{|l|}{ A. Butanol stress } \\
\hline & \multicolumn{2}{|c|}{ Low BuOH } & \multicolumn{2}{|c|}{ Med BuOH } & \multicolumn{2}{|c|}{ High BuOH } \\
\hline \multirow[t]{2}{*}{ Total proteins used for DE analysis } & 243 & & 280 & & 222 & \\
\hline & Up & Down & Up & Down & Up & Down \\
\hline DE proteins (FDR $5 \%$ ) & 23 & 40 & 20 & 63 & 53 & 63 \\
\hline DE proteins (FDR $5 \%$, fold change $\geq 2.0$ ) & 15 & 33 & 20 & 56 & 34 & 41 \\
\hline Proteins expressed only under stress & \multicolumn{2}{|l|}{37} & \multicolumn{2}{|l|}{59} & \multicolumn{2}{|l|}{25} \\
\hline Proteins expressed only under non-stress control & \multicolumn{6}{|l|}{44} \\
\hline \multicolumn{7}{|l|}{ B. Butyrate stress } \\
\hline & \multicolumn{2}{|c|}{ Low BA } & \multicolumn{2}{|c|}{ Med BA } & \multicolumn{2}{|c|}{ High BA } \\
\hline \multirow[t]{2}{*}{ Total proteins used for DE analysis } & \multicolumn{2}{|l|}{337} & \multicolumn{2}{|c|}{344} & \multicolumn{2}{|l|}{357} \\
\hline & Up & Down & Up & Down & Up & Down \\
\hline DE proteins (FDR $5 \%$ ) & 54 & 19 & 60 & 41 & 68 & 32 \\
\hline DE proteins (FDR $5 \%$, fold change $\geq 2.0$ ) & 38 & 17 & 38 & 26 & 36 & 22 \\
\hline Proteins expressed only under stress & \multicolumn{2}{|l|}{43} & \multicolumn{2}{|l|}{70} & \multicolumn{2}{|l|}{61} \\
\hline Proteins expressed only under non-stress control & \multicolumn{6}{|l|}{67} \\
\hline
\end{tabular}
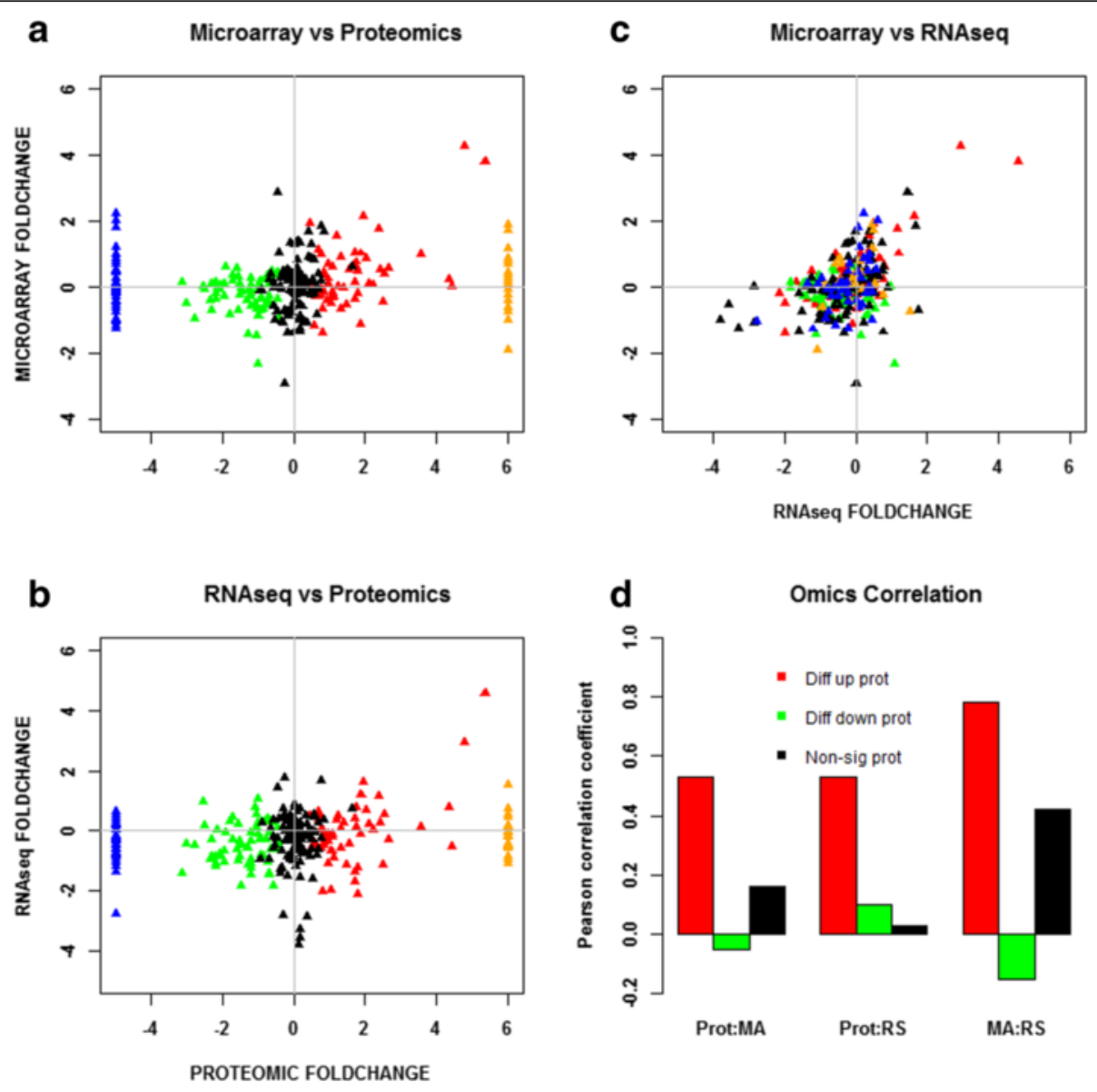

Fig. 4 Comparison and correlation between proteomic and transcriptomic data under high butanol stress. a Microarray versus proteomic comparison. b RNAseq versus proteomic comparison. c Microarray versus RNAseq comparison. $\mathbf{d}$ Pearson correlation. All significant expressions are with respect to proteomic data only. Red: differentially upregulated proteins; green: differentially downregulated proteins; black: non-significant proteins; blue: proteins expressed only under non-stress control; orange: proteins expressed only under stress 


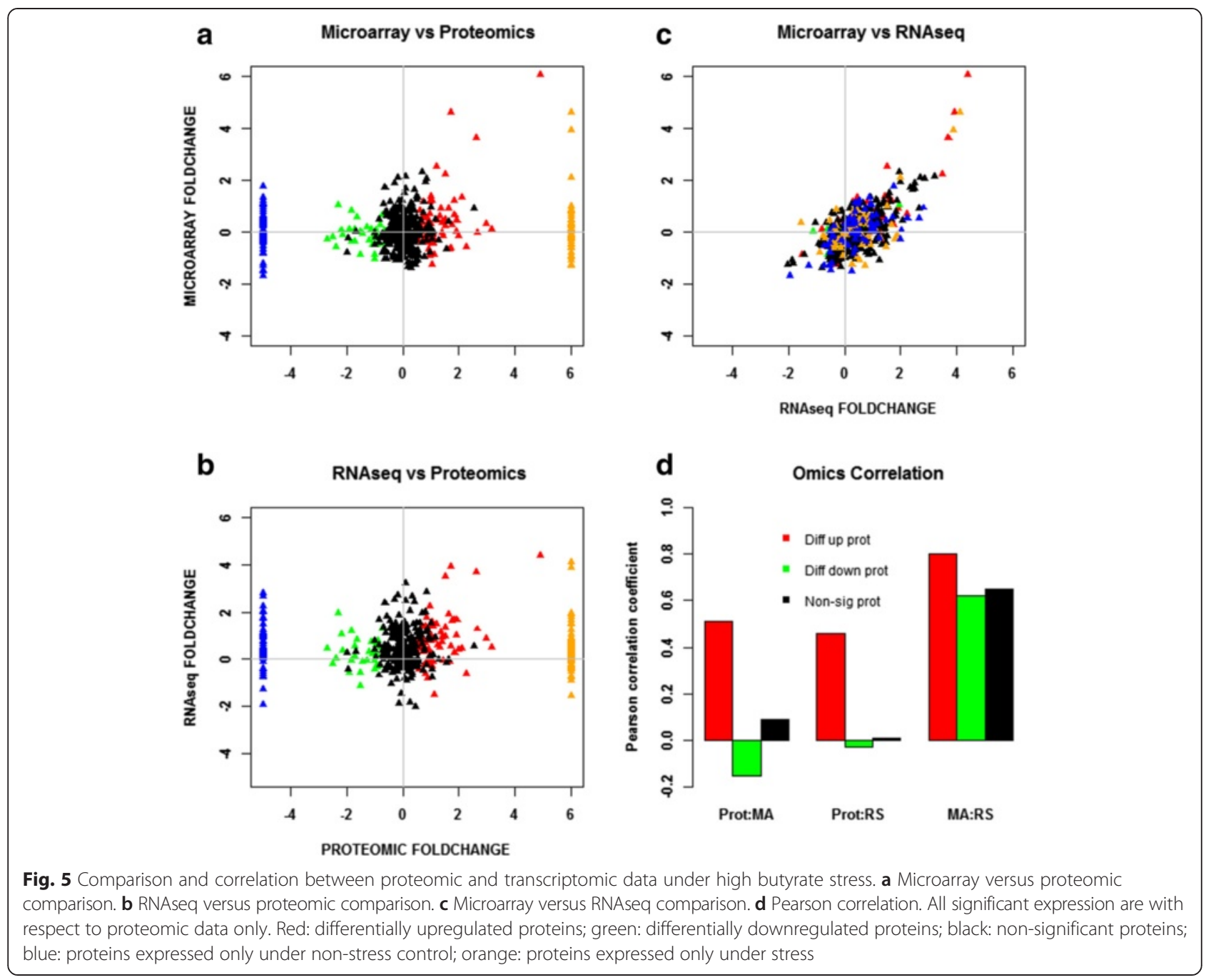

agreement or not between mRNA and protein levels. The most interesting ones are the set of genes/proteins that are not in agreement between the two sets and are also important to physiology. In addition to differential expression, it is useful to also display a metric of a

Table 2 Key proteins expressed only under butanol stress condition but not under non-stress control condition

\begin{tabular}{ll}
\hline Proteins & Function \\
\hline CAC1281 - GrpE & Heat shock response \\
CAC1283 - DnaJ & Heat shock response \\
$\begin{array}{l}\text { CAC0381 - methyl-accepting } \\
\text { chemotaxis protein }\end{array}$ & Chemotaxis \\
$\begin{array}{l}\text { CAC0433 - methyl-accepting } \\
\text { chemotaxis protein }\end{array}$ & Chemotaxis \\
$\begin{array}{l}\text { CAC0909 - methyl-accepting } \\
\text { chemotaxis protein }\end{array}$ & Chemotaxis \\
$\begin{array}{l}\text { CAC3545 - methyl-accepting } \\
\text { chemotaxis protein }\end{array}$ & Chemotaxis \\
CAC0653 - response regulator & \\
\hline
\end{tabular}

protein's or mRNA's expression level, which provides additional information for proteins/genes found expressed or differentially expressed under one condition but not in another, or at one level (protein or mRNA) but not the other. To this effect, as we have shown previously $[15,13]$ we employed the blue plots (heat maps), which display the relative abundance of a protein or mRNA with respect to total protein or mRNA

Table 3 Key proteins expressed only under butyrate stress condition but not under non-stress control condition. A heat map for the expression of these proteins is shown in Additional file 1: Figure S8.

\begin{tabular}{ll}
\hline Proteins & Function \\
\hline CAC3190 - Yacl & Heat shock response \\
CAC3191 - YacH & Heat shock response \\
CAC0083 - UV resistance protein & Stress response \\
CAC1412 - PemK (MazF/MazE) family regulator & Transcriptional regulator \\
CAC2215 - FliY & Flagellar motor protein \\
CAC2224 - CheW & Chemotaxis \\
\hline
\end{tabular}


level for given sample and time point. This relative abundance is displayed in percentile between 0 and 100 for the least and most abundant protein or mRNA, respectively. Twenty-three percent (102) and $31.5 \%$ (186) of the proteins detected under butanol and butyrate stress, respectively, displayed clearly opposite patterns from the corresponding mRNA expression patterns. These conservative estimates represent a surprisingly large fraction of the expressed proteome and could not have been anticipated from previously published studies.

Among the proteins that were found to be expressed only under the control conditions and not under butanol stress (despite good mRNA-level expression as displayed in the blue heat maps), three proteins, coded by genes CAC0943-hisE, CAC0936-hisG, and CAC2065-deoB (Fig. 2b), are involved in histidine/purine metabolism. These three mRNAs were differentially upregulated, but no protein was detected under butanol stress. HisE and HisG encode for the protein product that catalyze the first two steps of histidine biosynthesis from PRPP (phosphoribosyl pyrophosphate), while deoB is involved in the generation of PRPP, a key metabolite precursor in histidine, purine, and pyrimidine metabolism. Other genes, which belong to this category, include two ribosomal proteins - CAC3115 (rpmD) and CAC1284 (prmA). Butanol stress inhibits cell growth, and it appears that a select set of ribosomal proteins are downregulated as a result of the severe inhibition of protein synthesis.

On the other hand, several proteins, which were transcriptionally downregulated, were found to be expressed under butanol stress but not under the control nonstress condition (despite good mRNA levels; blue heat map), i.e., they appear to be upregulated under butanol stress (Fig. 2c). Some of them, with known biological significance to stress response and/or cellular metabolism, include three ribosomal proteins (CAC1259-rpmA, CAC1733-rpmB, and CAC1274-rpsT), a transcriptional elongation factor, greA (CAC2430), a peptide chain release factor (CAC0630), and a hypothetical protein (CAC2366). These data and the data in the previous paragraph suggest that a different set of proteins is engaged for protein synthesis under butanol stress. Some of the other proteins in this group include $\mathrm{ftsA}$ (CAC1692), a cell division protein; mutS1 (CAC2340) recombination and DNA strand exchange inhibitor protein; and gyrA (CAC0007) DNA gyrase which are involved in cell division and DNA replication and/or transcription. These findings suggest that the cells are using posttranscriptional regulation to upregulate proteins necessary for repairing DNA damaged by the butanol insult, and continue cell division despite the severe, overall, growth inhibition. Butanol stress has a strong negative effect on membrane functionality as it affects the membrane fluidity [20], which in turn affects membrane transport and the transmembrane potential and $\Delta \mathrm{pH}[21,22]$. Several $\mathrm{ABC}$ transporters and permeases (Fig. 2c) (CAC3262, CAC0662, CAC0272, CAC0570, CAC0108, CAC0107) for the transport of sugars, amino acids, and peptides were found to be translated under butanol stress but not under the control condition. These data suggest that the cells upregulate the expression of select membrane proteins, despite their transcriptional downregulation, aiming to deal with the chaotropic effect of butanol that inhibits membrane functions.

Figure $2 \mathrm{~d}$ summarizes the proteins that were upregulated under stress. There was a small subset of proteins that were not in agreement with the transcriptional data, i.e., they were translationally upregulated from, overall, differentially downregulated mRNAs. These included several proteins from carbohydrate and energy metabolism, cell division (ftsZ), and ribosomal proteins (CAC1787, CAC3105, CAC3147, CAC3132). Again, these data suggest that the cells upregulate the translation of select sets of proteins aiming to ameliorate the inhibitory impact of butanol and despite the downregulation of these transcripts.

Focusing next to butyrate stress, several genes/proteins showed a disagreement between protein and mRNA expression patterns (Fig. 3). Among the proteins that were detected only under butyrate stress but not under control conditions (Fig. 3b), despite good mRNA levels (blue heat map), are proteins involved in several stress response pathways. These include proteins involved in DNA damage, repair, and replication (CAC3723 (ssb) - single-strand DNA binding protein); stress-related protease for protein quality control (IonA) and peptide chain release factor (CAC0630); cell division (Maf - septum formation protein;

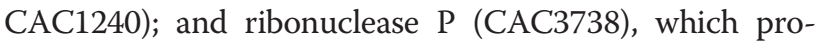
cesses tRNAs and possibly sRNAs (Fig. 3b). Unlike butanol stress, proteins involved in histidine (HisZ) and PRPP metabolism (CAC0819, pyrE) were found to be upregulated (Fig. 3b), despite downregulated transcript levels. The histidine/purine metabolism involving PRPP displays opposite behavior in comparison to butanol stress. Furthermore, several ribosomal proteins and proteins of amino acid metabolism, such as lysine metabolism (Fig. 3c), were found to be upregulated at protein level despite lower transcript levels. Proteins involved in DNA replication, DnaA and DnaN, and fatty acid metabolism FabH and FabD (involved in initiation and elongation) were not detected under butyrate stress in comparison to the control condition (Fig. 3e), despite higher amounts of transcript (blue heat map), likely reflecting lower DNA and fatty acid biosynthesis rates under butyrate stress. In the same category (expressed under control but not under butyrate stress, despite good mRNA levels of expression; Fig. 3e) is CAC2889, the only annotated for the hexameric transcription 
termination factor Rho, with several additional roles in transcription and translation recently added to its repertoire, including the premature termination and degradation of spurious transcripts [23]. In E. coli and other well-studied model prokaryotes, Rho is apparently responsible for the termination of about half of the coded transcripts. This would suggest that, under butyrate stress, mRNAs dependent on Rho for termination are affected and probably improperly processed or unstable, thus resulting in massive and global changes in the RNome under butyrate stress as seems to be suggested by the data of Figs. 1, 2 and 3. Of note is that both the protein and the transcripts of two other transcriptional termination proteins (CAC3216-Mfd and CAC1799-NusA; Fig. 3d) that may offer alternate mRNA termination mechanisms [24] are also downregulated under butyrate stress.

Finally, we would like to draw attention to the signal recognition particle (SRP) GTPase (Ffh, coded by CAC1752), which, together with a small RNA noncoding RNA (the SRP-RNA), creates SRP. The SRP translocates the ribosomes to synthesize proteins in the membrane using the membrane-associated SRP receptor, SR, coded by fts $Y$ (CAC1754). In our previous transcriptomic study [15], we found that the SRP-RNA was upregulated under butyrate stress. The transcriptomic data show low expression of ffh and ftsY, but the proteomic data revealed stress-specific expression of Ffh (Fig. 3b) under high butyrate stress. Surprisingly, the FtsY protein was not detected under butyrate stress although it was expressed (detected) at the control non-stress condition (Fig. 3e). These data may reflect the cell's need to synthesize at higher rates a set of membrane proteins to ameliorate butyrate toxicity.

To sum this section, a first comparative analysis of the differential expression of proteins and their corresponding mRNA revealed complex regulation at post-transcriptional and/or translational levels of several pathways and programs of stress physiology. Those included programs for amino acid and nucleic metabolism; DNA replication, repair, and damage; the transcription and translation machinery, including transcriptional termination; as well the SRP-system proteins that could not have been detected using of only one type of omic data.

\section{Probing protein- versus mRNA-level expression differences further}

To further detail the extent to which the protein and mRNA data agree or disagree, and to bring the RNAseqbased mRNA data into the analysis, two-way comparisons of proteomic, microarray, and RNAseq data are presented in $x-y$ scatter plots by grouping the proteins into five categories based on their pattern of expression: differentially upregulated, differentially downregulated, non-significant, expressed only under stress, and expressed only under control. For the last two groups, since expression of the proteins was detected only under one condition, as stated above, these two sets were assigned the maximum value observed for differentially (6.0) upor downregulated (-5.0) proteins (Figs. 4 and 5) and are discussed separately. Pearson correlation coefficient among the proteomic, microarray, and RNAseq datasets were calculated for each of the three categories (differentially upregulated, differentially downregulated, and non-significant; due to the lack of standard deviation, Pearson correlation cannot be calculated for the last two categories for the proteins that were either expressed only under stress or only under control nonstress condition) and between the two transcriptomic datasets (microarray and RNAseq). These comparisons are summarized in Fig. 4 for high butanol and in Fig. 5 for high butyrate stress, respectively. High stress levels overall appear to accentuate the distinct features of each stress condition. The corresponding plots for low and medium stress levels are presented as Additional file 1: (Figures S3-S6). Overall, the correlation between the two transcriptomic datasets is far superior to the proteomic-transcriptomic comparisons. The microarray versus RNAseq transcriptomic comparison shows extremely high correlation among the differentially upregulated genes/proteins (Figs. 4 and 5) but a lower correlation for the genes/proteins that were differentially downregulated or non-significantly expressed for butanol stress (Fig. 4). These disagreements arise due to the differences in technology between microarray and RNAseq along with transcript abundance and amplification of transcripts/cDNA during RNAseq library preparation [25].

A high correlation between mRNA and protein levels was observed among the differentially upregulated proteins and a low correlation between mRNA and protein level for the differentially downregulated and nonsignificantly regulated proteins. As discussed above, the majority of proteins belonging to the differentially upregulated group belonged to proteins of the post-translational modification, protein turnover, and chaperone systems. Differentially downregulated proteins have the lowest correlation between proteomic and transcriptomic data. This lack of correlation can be attributed to two key factors: inefficiency of the translational machinery under stress and post-transcriptional regulation of the transcripts, such as by regulatory non-coding small RNAs (sRNAs), which have been identified to be involved in this stress response [15]. We note that issues of mRNA and protein stability and degradation cannot be responsible for the observed differences as these two processes are already taken into account in the temporal "snapshots" of these omic data (Figs. 2 and 3). To further probe the basis for these differences, we examined the data from the 
regulon and program point of view, as well as from the point of view of the structural features of the corresponding mRNAs. The former would argue for an evolutionary basis for the observed disagreements between protein and mRNA levels, while the latter could explain how these differences can be explained by molecular regulation based on mRNA features. First, we analyzed key regulons and programs that likely play an important role under stress in this organism $[12,6,17,18,14,16]$.

\section{The stress proteome versus transcriptome of key regulons and the translation program HrCA and CtsR regulons}

The HrcA and CtsR regulons are two core stressresponsive regulons involved in the canonical stress or heat shock (HSP) response. These two regulons were precisely identified and detailed based on transcriptomic data and bioinformatics analyses [16]. The HrcA regulon, which consists of eight genes in four operons, was transcriptionally (based on both microarray and RNAseq data) strongly upregulated (Fig. 6a, b) in response to both stresses. The proteomic data show that, under stress, and especially butanol stress, these upregulated transcripts were overall poorly translated or that these proteins were unstable. Under butyrate stress, proteins for six of the eight HrcA genes were detected, three of which showed good correlation between mRNA and protein level. Under butanol stress, five of the eight proteins were detected and two to three showed reasonable correlation with mRNA patterns. GrpE and DnaJ were detected as expressed only under butanol stress but not under control conditions (Fig. 6a).

The CtsR regulon consists of 11 genes (Fig. 6c, d), which were highly upregulated at the mRNA level, under both butanol and butyrate stress. Seven and five of the corresponding proteins were detected under butyrate and butanol stress, respectively. Fewer among those showed any reasonable agreement with mRNA patterns. While the proteins of the two transcriptional regulators (HrcA and CtsR) were not sufficiently abundant to be detected by the proteome method used but based on the current and prior transcriptomic studies $[12,6,7,13-$ 16], one would have expected the proteins (heat shock and general stress proteins) regulated by these two transcriptional regulators to be detected under stress due to their stress-induced expression. Some of the observed patterns are also surprising for additional reasons. For example, YacI and $\mathrm{YacH}$, two genes in the $\mathrm{ClpC}$ operon, were detected as expressed only under high butyrate stress and not at all under butanol stress. In contrast, $\mathrm{ClpC}$ was detected as expressed under most stress conditions. We note that the two regulons share a few common members, notably HrcA, GrpE, DnaK, and DnaJ, which are located in the same genomic locus and organized in operons that may share common regulatory features [16]. Still, the protein patterns detected here are very different among these proteins and especially so between the two stress conditions. These data suggest strong translational regulation or protein instability for the CtsR and HrcA regulon proteins.

\section{The Rex regulon is important in the response to butyrate but not butanol stress}

Rex (CAC2713) is the redox sensor transcription (repressor) factor. Its regulon in $C$. acetobutylicum was identified using phylogenetic foot printing analysis [16]. The Rex regulon plays an important role in regulating the overall redox balance, NADH/NAD ${ }^{+}$levels, ATP synthesis, and electron transport, thus regulating the central carbon and energy metabolism, and is especially important in solventogenic clostridia as it has been shown to regulate the shift from acidogenesis to solventogenesis [26].

Both the rex transcript and Rex protein were found to be downregulated under butyrate stress (Fig. 6e), thus displaying good correlation between mRNA and protein levels. We were happily surprised to be able to detect Rex at the protein level, given that it is a regulator typically expressed at lower levels. Under butyrate stress, although only 20 of the 33 proteins of the Rex regulon were identified by our proteome method (Fig. 6e), there was overall in good correlation between protein and transcript levels (based on both the RNAseq and microarray data). Notably, the proteins/genes involved in ATP synthesis (CAC2864-CAC2871), electron transport (EtfA, EtfB), butyrate production (Thl, Crt, Bcd), butyrate assimilation to form butanol (AdhE1/Aad), amino acid metabolism (aminotransferase and SerA), and carbon and energy metabolism (GapC, aldolase) displayed an overall good correlation between mRNA and protein levels. It is interesting to note that although all three genes (namely the genes the sol operon (adhE1/aad-ctfA$c t f B)$ ) involved in solvent production were upregulated at the mRNA level, the protein of only the first gene, aad, was detectable under butyrate stress and found to be also upregulated. This would suggest preferential translation of selected genes in an operon under stress apparently due to post-transcriptional regulation. As shown from the physiological metabolite data (Additional file 1: Figure S1), the cultures stressed with butyrate produced more butanol with corresponding increase in butyrate stress but on the other hand, acetone production decreased with increasing in butyrate stress.

Unlike the butyrate stress, the rex gene was not affected by butanol stress, and its protein did not display a clear pattern of expression. It is not surprising then that 


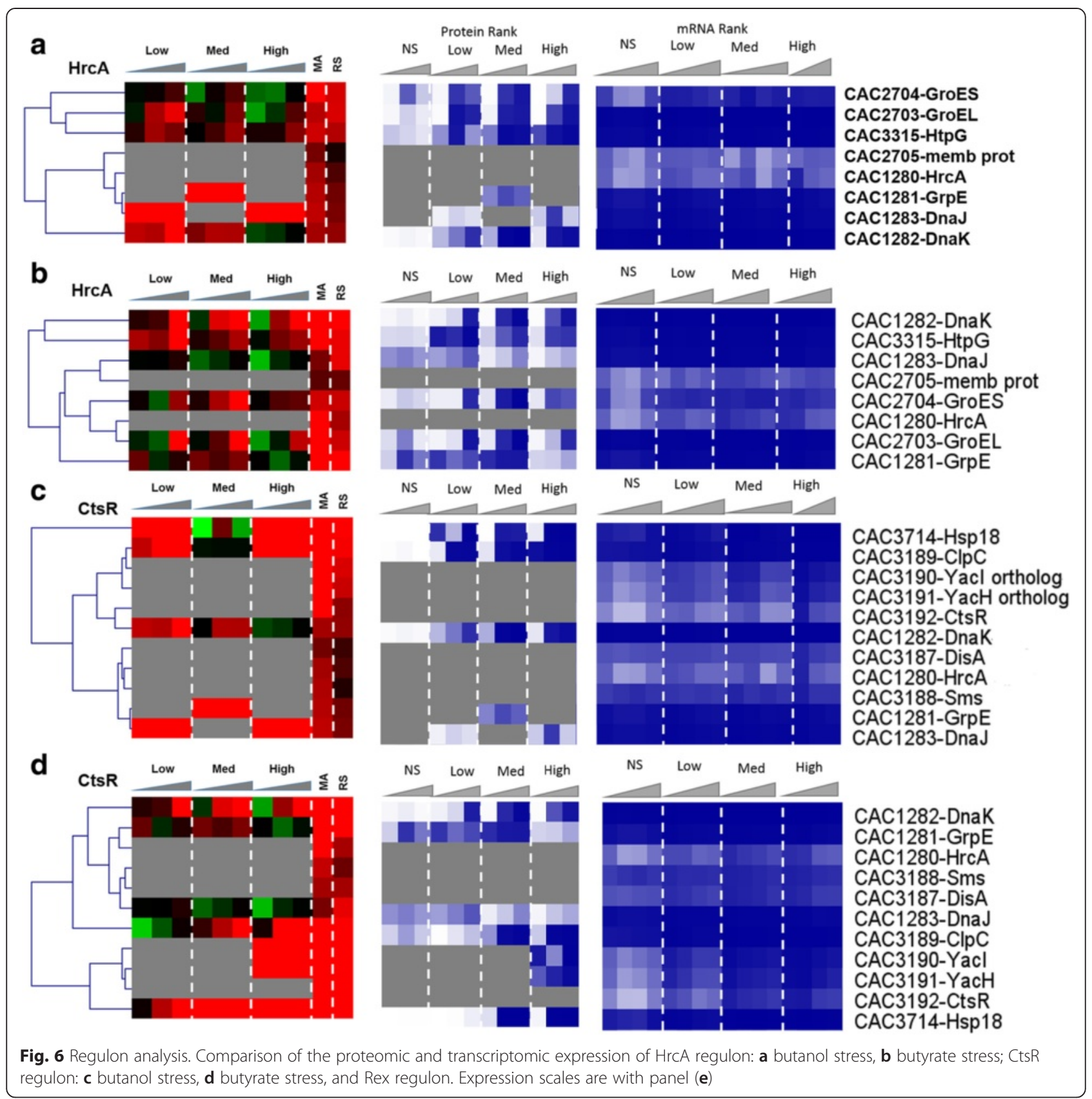

fewer proteins (14) of the Rex regulon were identified at the protein level under butanol stress, and that there was a poor correlation between protein and mRNA levels. The only interesting observation is that although all three transcripts of the sol operon were upregulated under butanol stress, none of the proteins was detectable by our method, again highlighting the importance of post-transcriptional regulation. We conclude that the Rex regulon has no important role in butanol stress.
Ribosomal and related proteins of the translation machinery proteins and the differential expression of leaderless transcripts

To assess the impact of metabolite stress on the translation machinery, the protein and transcripts levels of all 74 annotated ribosomal proteins and seven accessory proteins were analyzed (Fig. 7). The accessory proteins include GTPases that are essential for the translational machinery. Twenty of the ribosomal proteins and four of the accessory proteins remained undetected 


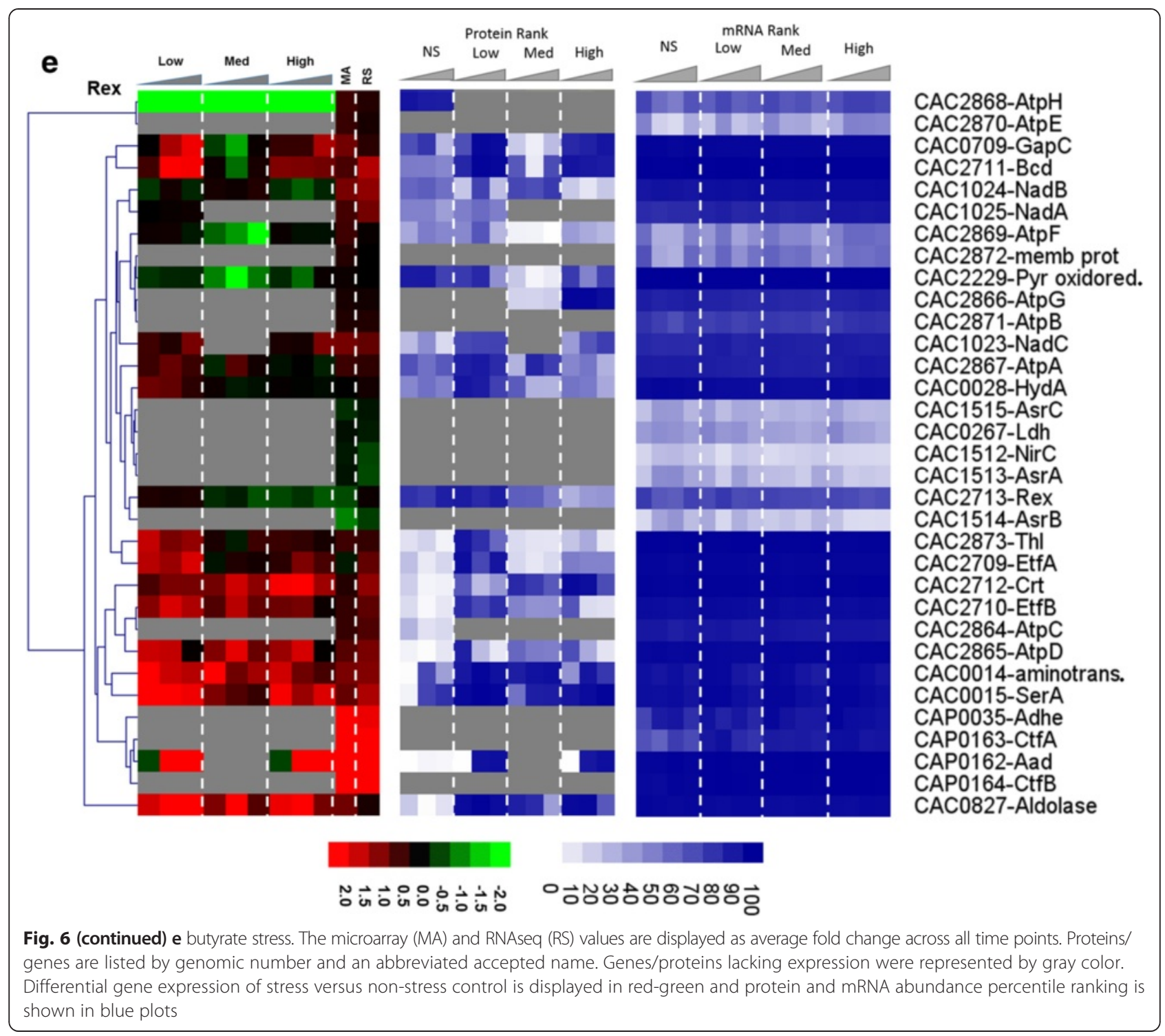

in the proteomic analysis under either butanol or butyrate stress. Among the detected 57 proteins, the majority was downregulated under butanol stress despite higher transcriptional levels. Overall, there was poor correlation between protein and mRNA levels for these proteins, with only a few exceptions. Under butyrate stress, which exhibited little or no inhibition of growth with respect to the non-stress control condition, a large fraction of the ribosomal proteins were upregulated and only a few downregulated. In contrast, under butanol stress, which leads to growth inhibition (Additional file 1: Figure S1), the majority of the ribosomal proteins were found to be downregulated, and this makes logical sense. It is interesting to note that a few ribosomal proteins were upregulated, including those coded by CAC1787, CAC3097, CAC3125, CAC3132, and CAC3147 thus suggesting that the cells may employ an alternate set of protein for protein synthesis under stress. Along the same lines, under butyrate stress, there were a few translation-related protein that were not only upregulated under some level of butyrate stress but those were proteins not detected under non-stress conditions and include CAC1803, CAC1284, and CAC1295. A specific mechanism that results in the use of an alternate translational machinery under stress in $E$. coli $[27,28]$ engages the toxinantitoxin (MazF-MazE) system that generates leaderless transcripts and a subpopulation of alternate ribosomes to translate the leaderless transcripts. In leaderless transcripts, the transcriptional start site and the translational start sites are the same, and thus they lack a ribosomal binding site, RBS. No other stress-specific mechanisms regarding changed ribosomal composition have been 


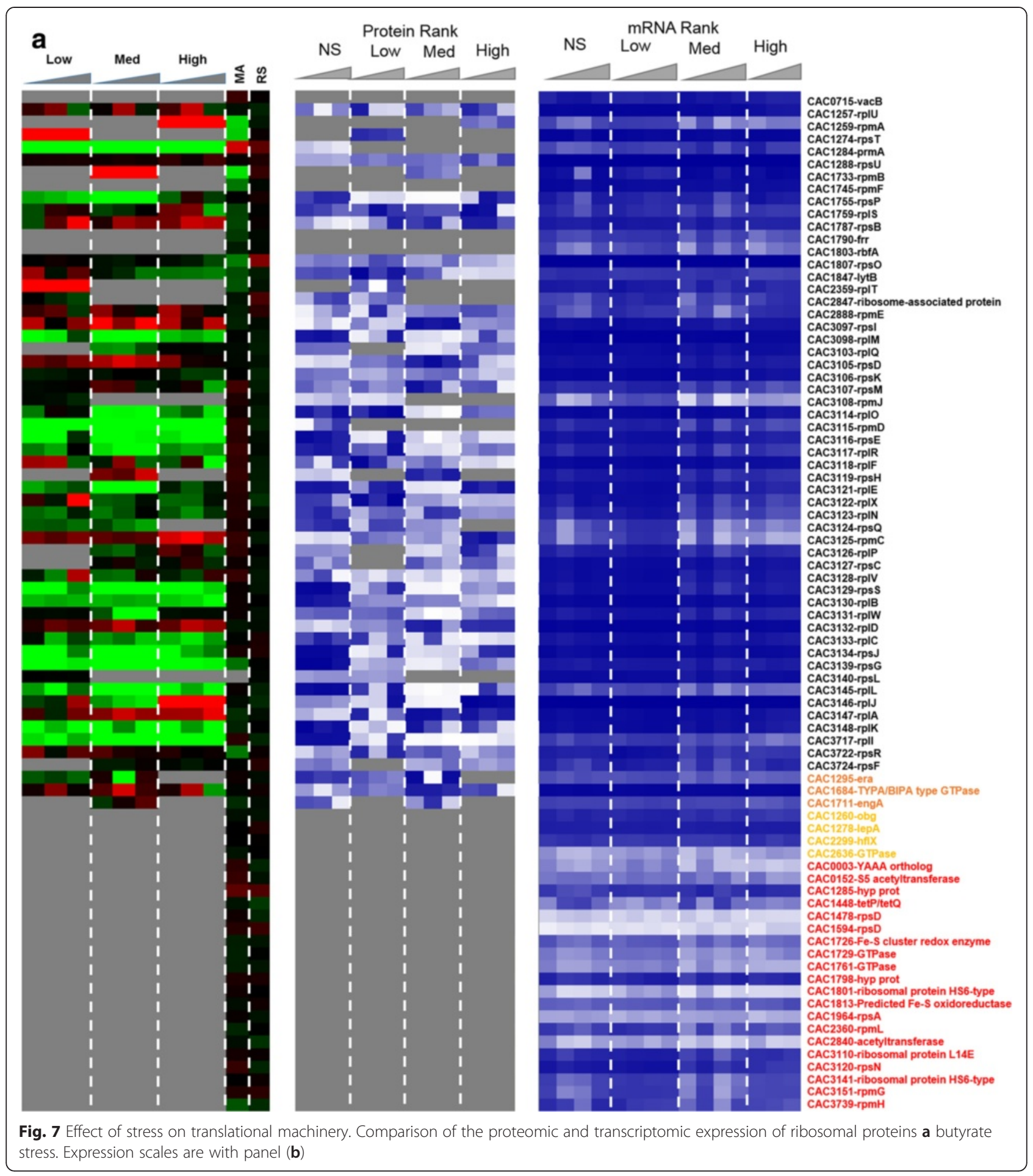

reported in the literature. This prompted us to examine the existence and possible differential transcription of leaderless transcripts.

Leaderless transcripts are either naturally coded on the chromosome as leaderless or are generated by some specific mechanism that trims canonical mRNAs that contain a RBS. Using the strand-specific RNAseq data, we identified 212 leaderless transcripts among the genes corresponding to the proteins identified by proteomic analysis. These leaderless transcripts are, by definition, either monocistronic operons or the first gene of a polycistronic operon, based on the transcriptional operon organization of C. acetobutylicum [29]. Further, we identified 102 transcripts under butanol stress and 120 


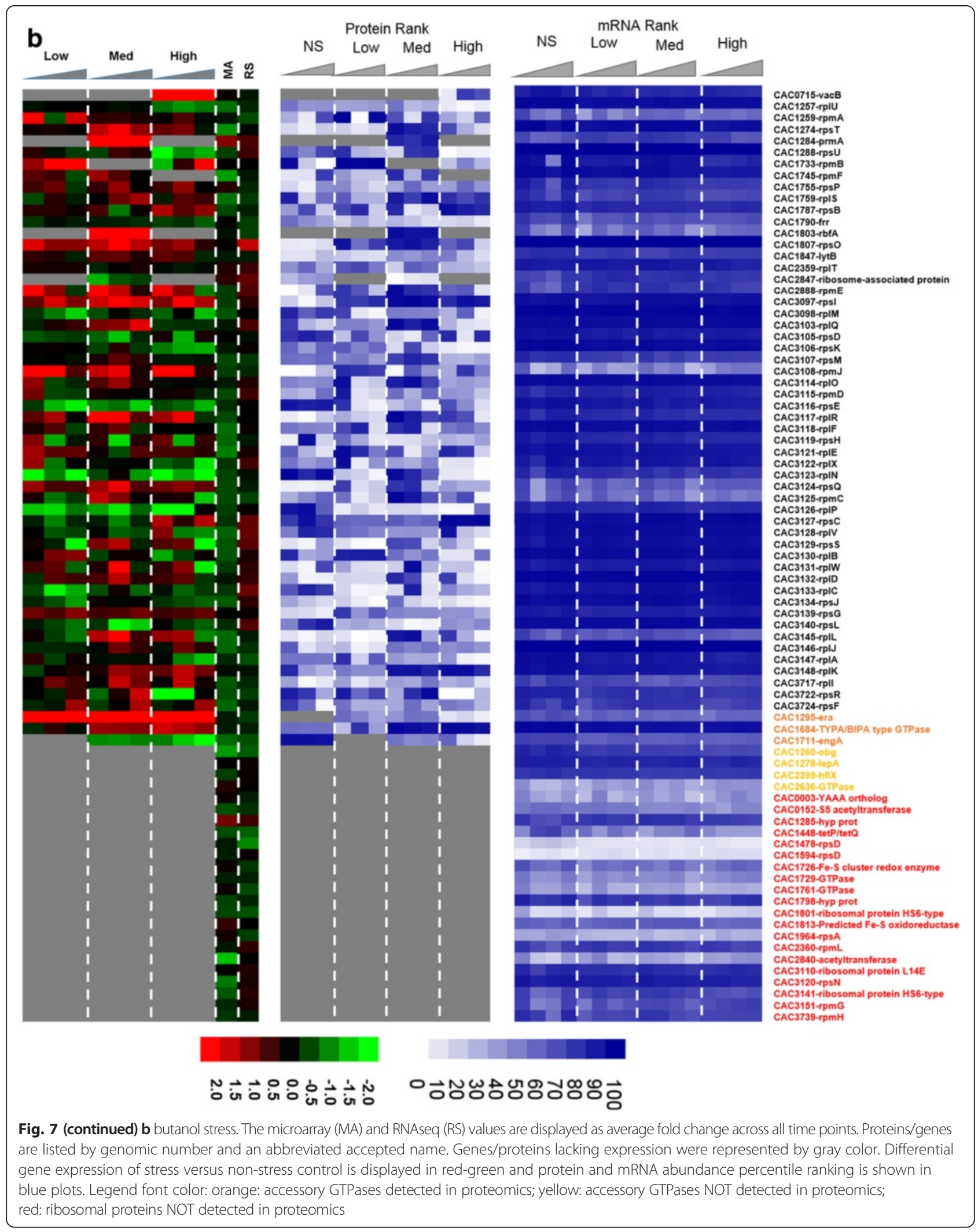


transcripts under butyrate stress, as being leaderless (Additional file 1: Figure S7). Among these leaderless transcripts, 22 and 37 corresponded to proteins that had a strong disagreement between proteins and mRNA levels (Figs. 2 and 3) under butanol and butyrate stress, respectively. Nine transcripts which had 5'UTR under non-stress condition were found to be leaderless under both butanol and butyrate stress. On the other hand, 28 and 29 leaderless transcripts under non-stress condition were found to contain $5{ }^{\prime}$ UTR with a RBS under butanol and butyrate stress. These results confirm the presence of leaderless transcripts in this organism along with differential transcriptional start sites under stress. Leaderless transcripts have been identified in several bacterial transcriptomes [30-33]. A general mechanism, identified in E. coli (but not in C. acetobutylicum yet) for the translation of leaderless transcripts, involves the initiation factors 2 (IF-2) and 3 (IF-3) [34], where a higher IF-2/IF-3 ratio would favor the transcription of leaderless transcripts, such as has been observed under a cold shock in E. coli [34]. The C. acetobutylicum IF-3 protein (CAC2361) is expressed well under no stress conditions but was undetectable under butanol stress (Fig. 2b), thus presumably leading to a higher IF2/IF-3 ratio. IF-2 (CAC1802) was downregulated under butyrate stress (Fig. 3d). In this light, we re-assessed possible roles of the downregulated ribosomal proteins (Fig. 7) in transcriptional elongation and termination. In $E$. coli, one well-known protein among those, S10 (coded by the CAC3134 (rps) gene in C. acetobutylicum) is part of a transcriptional antitermination system with the transcriptional elongation factors NusA and NusG [24] (these are coded by CAC1799 and CAC3149, respectively, in C. acetobutylicum). This system interacts with the Rho protein to control intragenic transcriptional Rho-dependent termination [23]. The systematic downregulation of the S10 protein under both butyrate and butanol stress suggests changes in transcriptional termination under stress, and this is further supported by the finding that the expression of the Rho protein (which plays also a role in the coupling between translation and transcription [23]) is strongly downregulated under butyrate stress as already discussed.

As discussed above, in addition to naturally coded leaderless transcripts, in E. coli, the MazE/MazF system has been found to generate leaderless transcripts and facilitate their translation by generating modified ribosomes $[27,28]$. An ortholog of the MazF-MazE system has been annotated in C. acetobutylicum (CAC0493CAC0494). These two genes do not display any changes at the transcriptional level and were not detected by our proteomic analysis, thus reflecting a low level of protein expression. Nevertheless, their role in clostridial stress response deserves further investigation. It is not unlikely, however, that other mechanisms exist to trim canonical mRNAs into leaderless transcripts.

\section{What drives the disagreement between mRNA and protein levels for such a large fraction of the genome? Mechanisms involving 5'UTR length and RBS strength}

A number of studies across different species and organisms have shown lack of good correlation between mRNA and protein levels [35-37]. Such lack of correlation could be explained by different mechanistic hypotheses all based on structural features of mRNAs that would enable complex physiological regulation, which here is stress-responsive regulation. mRNA translation efficiency can be affected by the strength of the ribosome binding site (RBS) [38], the use of rare codons in the mRNA [39, 31], or regulation of the translationinitiation process [31], such as accessibility of the mRNA by the translation proteins as might be affected by special features of the $5^{\prime}$ untranslated region ( $5^{\prime}$ UTR) of the mRNA [40]. Special features of the $5^{\prime}$ UTR could include self-bending of the 5'UTR preventing accessibility to the RBS, thus requiring specific sRNAs to prevent bending/looping and thus allow translation [41]. Other regulation could include binding of specific sRNAs near the RBS thus preventing translation [42]. Transcripts engaging such regulation are typically characterized by longer 5'UTRs.

Based on these mechanistic possibilities, we probed three hypotheses as discussed below. This required that we compute the length of the 5'UTR of all transcripts and also of the RBS strength. Transcriptional start sites (TSSs) along with 5'UTRs were determined using our strand-specific RNAseq data (see Materials and methods for more details). A total of $3895^{\prime}$ UTRs were determined for transcripts encoded in the chromosome, and 24 5'UTRs were determined for the transcripts in the megaplasmid pSOL1. In C. acetobutylicum, the median length of the 5'UTR is 42 for the first genes of an operon, which is similar to the numbers reported in other bacterial species [30, 43]. Thus, in view of the existence of many leaderless transcripts, 5'UTRs longer than 42 would likely contain regulatory elements that could affect mRNA stability and translation. We calculated RBS strength using Prodigal [44], a software that determines translational start sites and assigns a score to the RBS based on the motif and the spacer, which is the distance between the RBS motif and the start codon. These scores varied between -18.92 for extremely weak or no RBS and 12.87 for strongest RBS. The average score for the RBS was 7.09. One hundred ninety-three genes were found by the Prodigal algorithm to contain no RBS. Thus, RBS strength values above 7 or 8 could be viewed as indicating strong translational possibilities. Next, we examined three mechanistic hypotheses. 
The first hypothesis is that there is a set of mRNAs, which is translated inefficiently due to regulation by stress-responsive mechanisms affecting the accessibility of the mRNA for protein translation, such as the need for sRNAs to enable translation [41, 15]. These mRNAs then should display an anti-correlation with high mRNAs but relatively low protein levels under all conditions alike, stressed or non-stressed control conditions. An analysis of the data based on this hypothesis led to a null set, indicating that mRNAs with such anticorrelation were not observed under all conditions in this study.

The second hypothesis is that there is a set of mRNAs, which are translated inefficiently as a result of regulation from sRNAs or other related mechanisms under stress but not under non-stress condition. These mRNAs should have an anti-correlation with high mRNA levels (fold change $>2.0$ ) and low protein levels. We limited the search to medium and high level of stress conditions. No such proteins were found under either butanol or butyrate stress. We modified this hypothesis as discussed in the next section.

Our third hypothesis is that there is a set of mRNAs that display extraordinarily high translation efficiency and/or protein stability under stress conditions with low mRNA levels but high protein levels. We discovered 11 and 12 proteins/genes (hypothesis 3 ) under butyrate and butanol stress, respectively (Tables 4 and 5 ). These proteins were found to be differentially expressed, but their corresponding transcripts were not differentially expressed; rather, they displayed non-significant differential expression. Among the 11 proteins identified under butyrate stress, four (CAC1393 - PurM from purine metabolism, CAC3713 - hypothetical protein, CAC3097 - RpsI ribosomal protein, CAC2641 - trigger factor) and five (CAC0897 - aro, CAC3171 - LeuB, CAC3243 - chemotaxis protein, CAC0827 - fructose bisphosphate aldolase, CAC0972 isocitrate dehydrogenase) proteins were found at medium and high stress, respectively, while two (CAC3598 rubrerythrin and CAC0316 - ArgF/I) were found under both medium and high stress. Similarly, among the 12 proteins identified under butanol stress, three (CAC2229 -

Table 4 Key genes with high protein levels from low mRNA levels-butyrate stress

\begin{tabular}{llll}
\hline Gene & Function & 5'UTR $^{\prime}$ & RBS score \\
\hline CAC3713 & Hypothetical protein & 96 & 12.87 \\
CAC2641 & Trigger factor & 33 & 12.87 \\
CAC0897 & Aro & 13 & 9.25 \\
CAC3171 & LeuB & 30 & 7.68 \\
CAC3243 & Chemotaxis protein & 15 & 12.38 \\
CAC3598 & Rubrerythrin & 96 & 12.87 \\
\hline
\end{tabular}

Genes are in italics to indicate their differential proteomic upregulation
Table 5 Key genes with high protein levels from non-significant mRNA levels-butanol stress

\begin{tabular}{llcc}
\hline Gene & Function & 5'UTR & RBS score \\
\hline CAC0116 & CODH $\beta$ subunit & 84 & 12.87 \\
CAC2229 & PFOR & 76 & 9.25 \\
CAC2333 & Spsl - dTDP-glucose pyrophosphorylase & 104 & 12.87 \\
CAP0165 & Adc - acetoacetate decarboxylase & 63 & 12.09 \\
CAC3171 & LeuB & 30 & 12.38 \\
CAC3598 & Rubrerythrin & 96 & 12.87 \\
\hline
\end{tabular}

Genes are in italics to indicate their differential proteomic upregulation

pyruvate:ferredoxin oxidoreductase, CAC0578 - MetH, CAC3392 - Bdh) and five (CAC2709 - EtfA, CAC0022 aspartate semialdehyde dehydrogenase, CAP0165 - Adc, CAC2333 - SpsI, CAC3146 - RplJ) were identified under medium and high stress, respectively, while four (CAC3171, CAC3598, CAC0116 - CODH $\beta$ subunit, and $\mathrm{CAC} 2710$ - EtfB) were found under both medium and high butanol stress. Examination of the 5'UTR from transcriptomic data and RBS strength scores (Tables 4 and 5) shows that virtually all genes have high (much above the average) RBS scores and about half of them has also long (much above the average) 5'UTRs, which could account for the high protein levels despite low mRNA levels. Notable among these proteins is rubrerythrin as discussed next.

Rubrerythrin has been reported to act as an oxidative stress response protein in C. acetobutylicum $[45,46]$ and other Clostridium species [47, 48]. Rubrerythrin is viewed as a scavenger of dioxygen by acting as electron transport intermediary [48, 49]. In C. acetobutylicum, there are two copies of the gene (CAC3597 and CAC3598) and three other proteins, with an identity of $50 \%$, namely, CAC2575, CAC2778, and CAC3018, which are also annotated as rubrerythrins. Expression from our proteomic data mapped with the two copies of CAC3597-CAC3598, forming an operon in an arrangement viewed as a gene duplication [46]. Our proteomic data show that it is upregulated an average of 16 -fold under butyrate stress, and up to 40 -folds under butanol stress (Figs. 2, 3, 4 and 5). This clearly suggests the possibility of post-transcriptional regulation and/or high protein stability. Furthermore, using our strand-specific RNAseq data, CAC3598, the first of the two rubrerythrin genes in the operon, was found to contain a long 5'UTR of 96 nucleotides (Table 3). RNA secondary structure (Additional file 1: Figure S9) of the 5'UTR on its own and 5'UTR with varied length of the ORF (40 bases, 84 bases, and the full ORF) displayed the presence of loop of the $5^{\prime}$ UTR and the protein coding region. The access to RBS progressively decreased and became more stable (denoted by an increase in the free energy) with an increase in the length of the ORF and 
hence preventing the binding of ribosomes for translation [50]. This inhibition is usually removed by the expression of a specific non-coding sRNA, as has been reported in other organism [51] and requires further investigation in C. acetobutylicum with respect to their stress-specific sRNome [15].

\section{Revising and revisiting hypotheses 2 and 3}

We revisited hypotheses 2 and 3 , by comparing the abundance of mRNA and protein. As hypotheses 2 and 3 compared differential expression of mRNAs and their corresponding proteins, the revised hypotheses compared the actual abundance of the mRNAs and proteins among the entire transcriptome and proteome at a given stress condition. These modified hypotheses are if low abundance mRNAs express highly abundant proteins or if highly abundant mRNAs have low expression of their encoded proteins. To examine these revised hypotheses, the average transcript abundance percentile ranking for all the identified proteome under each stress condition was plotted against the average protein abundance percentile ranking (Fig. 8 and Additional file 1: Figures S10-S13).
Protein expressed from transcripts with a percentile ranking as low as 2 was observed under butanol stress while the minimum average transcript abundance percentile ranking of the observed proteins under butyrate stress was 10. If a linear relationship between the mRNA and protein level were to exist, the abundance ranking plot would contain the differentially upregulated proteins at the top right corner with highly abundant transcripts and the differentially downregulated proteins on the bottom left with their low abundance transcripts. Nevertheless, this is not true, as the relationship between mRNA and protein is not linear. Hatzimanikatis and Lee have reported that the non-linear relationship between mRNA and protein level is driven by several factors such as mRNA stability and degradation, translation efficiency for a given protein along with the presence of posttranscriptional regulation [52]. Hence, we focused on those proteins that displayed such non-linear relationships (Fig. 8) and analyzed them further. These analyses were limited to medium butyrate stress and high butanol stress as these conditions showed more such interesting discordances.
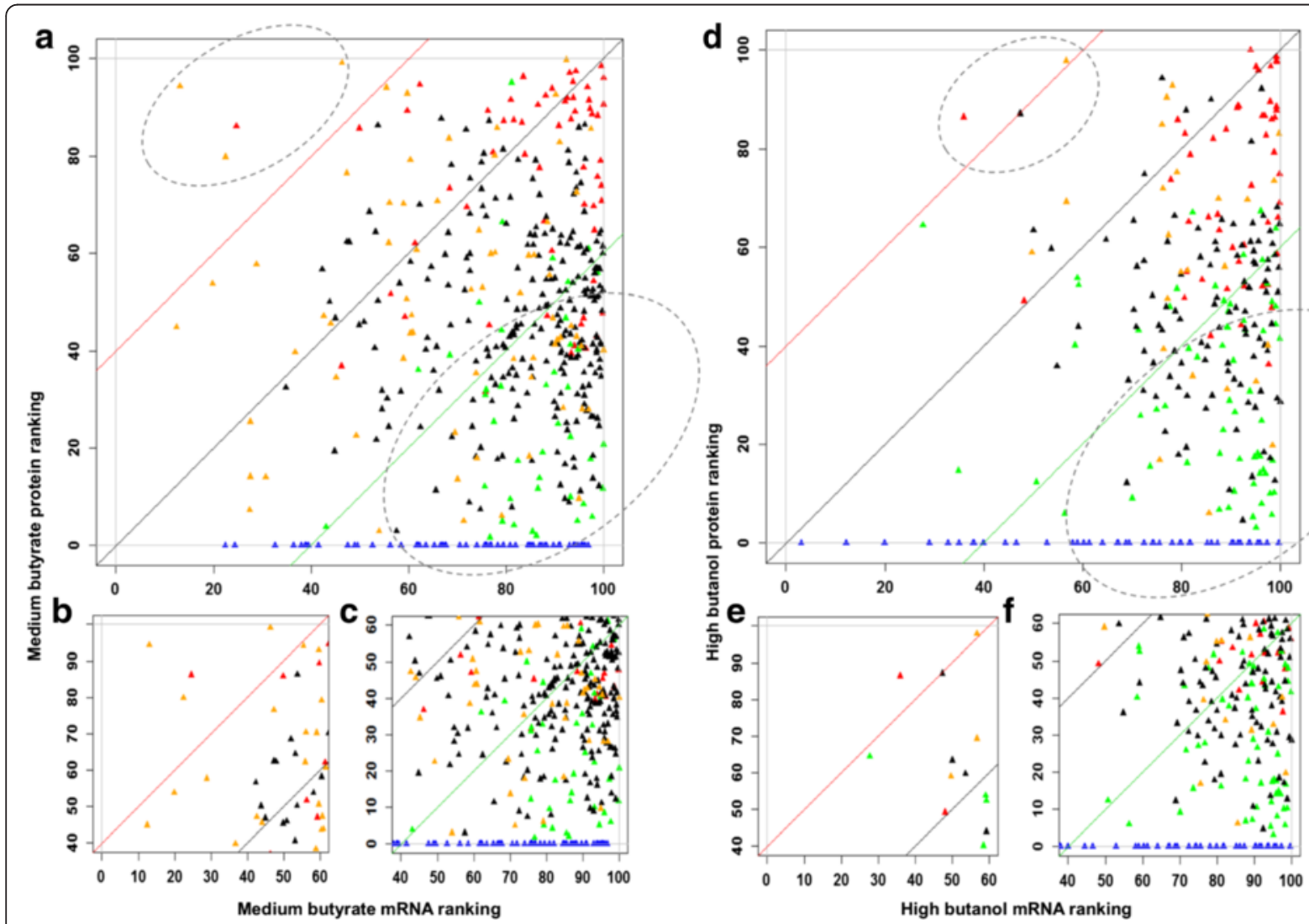

Fig. 8 Comparison of the transcript-protein abundance percentile ranking. $(\mathbf{a}, \mathbf{b}, \mathbf{c})$ medium butyrate stress and $(\mathbf{d}, \mathbf{e}, \mathbf{f})$ high butanol stress. X-axis represents the average percentile transcript (RNAseq) abundance ranking under stress, and y-axis represents average percentile transcript abundance ranking under non-stress control. Red: differentially upregulated proteins; green: differentially downregulated proteins; black: non-significant proteins; blue: proteins expressed only under non-stress control; orange: proteins expressed only under stress 
Table 6 Genes with less abundant ( $<60$ percentile rank) mRNA and highly abundant ( $>80$ percentile rank) proteins-butyrate stress

\begin{tabular}{llc}
\hline Gene & Function & RBS score \\
\hline CAC1425 $^{\text {a }}$ & Dut - dUTP hydrolase & 11.11 \\
CAC3654 $^{\text {a }}$ & Heavy metal binding & 12.38 \\
CAC0819 $^{\text {a }}$ & PRPP syn. & 12.38 \\
CAC3217 & DNA binding protein & 12.87 \\
\hline
\end{tabular}

Genes are in italics to indicate their differential proteomic upregulation, while bold represents proteins that were expressed only under butyrate stress and lacked expression under control condition

${ }^{a} 5$ 'UTR was not determined due to lack of sufficient data (reads)

${ }^{b}$ CAC3211 had a 5'UTR of 19 under butyrate stress but was leaderless under non-stress condition

Under medium butyrate stress, four proteins (Table 6) had low mRNA abundance ranking $(<60)$ but had higher protein percentile ranking $(>80)$. CAC3211 was differentially upregulated, while the other three proteins were found to be expressed only under butyrate stress. As these proteins were expressed from relatively less abundant mRNAs, their corresponding ribosome binding sites (RBSs) were analyzed. All the four genes, CAC3211, CAC3654, CAC0819, and CAC1425, contained high RBS scores near the upper limit of 12.87 , namely 12.87 , $12.38,12.38$, and 11.11 , respectively. The presence of such strong RBSs in these proteins can explain the high protein levels despite low mRNA levels. Similarly under high butanol stress (Fig. 8, Table 7), CAC0108 (sulfate ABD transporter permease), CAC0091 - IlvC, and CAC1301 - hypothetical protein had higher protein expression and lower mRNA levels as well as very high RBS scores at or near the upper limit of 12.87 .

Correspondingly, a large number of downregulated proteins, proteins that were expressed under stress and proteins that were expressed only under control conditions, displayed high transcript abundances but had lower levels of proteins. Many of these genes had transcripts with leaderless sequences leading to poor translation.

Table 7 Genes with less abundant mRNA ( $<60$ percentile rank) and highly abundant proteins ( $>80$ percentile rank) - butanol stress

\begin{tabular}{llc}
\hline Gene & Function & RBS score \\
\hline CAC0108 $^{\text {a }}$ & ABC transporter & 12.87 \\
CAC0091 $^{\text {b }}$ & IlvC & 12.38 \\
CAC1301 $^{\text {a }}$ & Hypothetical & 9.25 \\
\hline
\end{tabular}

Genes are in italics to indicate their differential proteomic upregulation, while bold represents proteins that were expressed only under butyrate stress and lacked expression under control condition. Bold italics represent non-significant protein expression

${ }^{a} 5^{\prime}$ UTR was not determined due to lack of sufficient data (reads) for CAC0108 and CAC1301

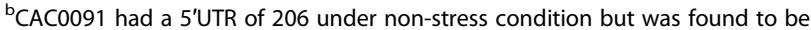
leaderless under butanol stress
Finally, the presence of long 5'UTRs for genes that were both transcriptionally and transnationally upregulated were found. One such protein is the Hsp18 containing a $5^{\prime}$ UTR of 100 bases, which may play a role in the stability of the transcript and its effective translation under stress conditions. It has a very strong RBS score of 12.87 and was upregulated at the protein level to a maximum of 109- and 111-fold under butyrate and butanol stress, respectively, with similar fold changes (100) reflected at the transcript level. The role of Hsp18 in stress response has been established from previous transcriptional studies on C. acetobutylicum $[12,6,7,53,17$, 16]. At the protein level, its expression was reported to increase during solventogenesis in comparison to acidogenesis $[54,55]$ and is also expressed at higher levels in a butanol hyper-tolerant mutant compared to the WT strain [55]. Our analysis here provides insight into a possible role of its long 5'UTR and strong RBS score in achieving these extraordinary high levels of protein upregulation.

\section{Discussion}

There have been nine, genome-scale proteomic studies on $C$. acetobutylicum, most of which were performed using two-dimensional gel electrophoresis combined with mass spectrometry (Table 8) [54-62]. Schaffer et al. (2002) investigated the proteins that were induced during the solventogenic phase of fermentation and identified 86 proteins (52 upregulated and 34 downregulated, fold change $\geq 2$ ) that had differential expression during solventogenesis [56]. Sullivan and Bennett (2006) analyzed over 200 spots in the WT strain and the Spo0A overexpression strain, for which they analyzed 23 proteins [57]. Among these 23 proteins identified, 22 were also identified in our study, most of which were found to be differentially expressed under stress. Apart from the identification of proteins with differential expression during different metabolic phases of growth and between strains, proteins with more than one spot were also reported, indicating possible post-translational modifications (PTMs; such as phosphorylation, acetylation, or glycosylation). Notably, DnaK, Hsp18, Adc, GroEL, Tpi, Bcd, and Chw16/17 were found to be present in two spots with identical molecular weights but different pI values $[57,56]$. Bai and Ji (2012) investigated the phosphoproteome of $C$. acetobutylicum and identified 61 proteins with phosphorylation on the $\mathrm{S} / \mathrm{T} / \mathrm{Y}$ residues, among which 57 proteins were identified in the stress proteome of the current study (56 proteins under butyrate stress and 54 proteins under butanol stress) [58]. Among these 57 phosphoproteins, 31 proteins were found to differentially expressed (FDR $5 \%$, fold change $\geq 2$ ) under stress with 17 and 21 proteins differentially expressed under butyrate and butanol stress, respectively. 
Table $\mathbf{8}$ Comparison and validation of the proteomic data with earlier reported proteomic work

\begin{tabular}{|c|c|c|c|c|c|}
\hline Work & Brief description & Proteins identified & $\begin{array}{l}\text { Proteins found in this } \\
\text { work (iTRAQ) }\end{array}$ & Key findings & $\begin{array}{l}\text { Comparison with } \\
\text { mRNA expression }\end{array}$ \\
\hline $\begin{array}{l}\text { Schaffer } \\
\text { et al. (2002) }\end{array}$ & Solventogenesis & 130 & $\begin{array}{l}\text { All } 130 \text { proteins have been } \\
\text { identified under butanol/ } \\
\text { butyrate stress }\end{array}$ & $\begin{array}{l}\text { Proteins involved in the } \\
\text { solventogenic pathway }\end{array}$ & $\begin{array}{l}\text { Northern analysis } \\
\text { (selected few) }\end{array}$ \\
\hline $\begin{array}{l}\text { Sullivan and } \\
\text { Bennett } \\
\text { (2006) }\end{array}$ & $\begin{array}{l}\text { Acidogenesis and } \\
\text { solventogenesis }\end{array}$ & 23 & $\begin{array}{l}22 \text { proteins were observed } \\
\text { in this study }\end{array}$ & $\begin{array}{l}\text { Proteins expressed during the } \\
\text { onset of solventogenesis }\end{array}$ & $\begin{array}{l}\text { With microarray } \\
\text { data from Tomas } \\
\text { and Alsaker (2004) }\end{array}$ \\
\hline $\begin{array}{l}\text { Mao et al. } \\
\text { (2010) }\end{array}$ & $\begin{array}{l}\text { Cytoplasmic proteins } \\
\text { (DSM } 1731 \text { and Rh8 } \\
\text { tolerant mutant) }\end{array}$ & 564 & $\begin{array}{l}86 \text { out of the } 102 \\
\text { differentially expressed } \\
\text { proteins were identified }\end{array}$ & $\begin{array}{l}\text { Proteins that play a role in } \\
\text { solvent toxicity tolerance }\end{array}$ & $\begin{array}{l}\text { qRT-PCR of } \\
\text { selected } \\
\text { differentially } \\
\text { expressed protein }\end{array}$ \\
\hline $\begin{array}{l}\text { Janssen } \\
\text { et al. (2010) }\end{array}$ & $\begin{array}{l}\text { Continuous culture of } \\
\text { WT in acidogenic and } \\
\text { solventogenic phases }\end{array}$ & $178+205$ & $\begin{array}{l}178 \text { acidogenic proteins } \\
\text { and } 205 \text { solventogenic } \\
\text { proteins }\end{array}$ & $\begin{array}{l}\text { Proteins that are expressed } \\
\text { under different metabolic } \\
\text { phases }\end{array}$ & DNA microarrays \\
\hline $\begin{array}{l}\text { Mao et al. } \\
\text { (2011) }\end{array}$ & $\begin{array}{l}\text { Membrane protein } \\
\text { DSM } 1731 \text { and } \mathrm{RH} 8 \\
\text { tolerant phenotype }\end{array}$ & 341 & $\begin{array}{l}23 \text { out of the } 33 \\
\text { differentially expressed } \\
\text { membrane proteins were } \\
\text { identified }\end{array}$ & $\begin{array}{l}\text { Membrane proteins that were } \\
\text { differentially expressed in the } \\
\text { butanol hyper-tolerant mutant }\end{array}$ & None \\
\hline $\begin{array}{l}\text { Sivagnanam } \\
\text { et al. (2011) }\end{array}$ & $\begin{array}{l}\text { CAC proteome under } \\
\text { glucose and xylose } \\
\text { utilization }\end{array}$ & 717 (glucose) 826 (xylose) & $\begin{array}{l}22 \text { of the } 23 \text { differentially } \\
\text { expressed proteins were } \\
\text { identified }\end{array}$ & $\begin{array}{l}\text { Proteins that correspond to } \\
\text { differential utilization of carbon } \\
\text { sources }\end{array}$ & None \\
\hline $\begin{array}{l}\text { Bai and Ji } \\
(2012)\end{array}$ & $\begin{array}{l}\text { Phophoproteome of } C \text {. } \\
\text { acetobutylicum }\end{array}$ & 61 phospho-proteins & $\begin{array}{l}57 / 61 \text { phosphor proteins } \\
\text { were also identified in our } \\
\text { work }\end{array}$ & $\begin{array}{l}\text { Proteins with post translational } \\
\text { modifications (PTMs) and their } \\
\text { role in stress response }\end{array}$ & None \\
\hline $\begin{array}{l}\text { Sivagnanam } \\
\text { et al. (2012) }\end{array}$ & $\begin{array}{l}\text { Protein interaction } \\
\text { network using STRING } \\
\text { and CYTOSCAPE }\end{array}$ & $\begin{array}{l}217 \text { proteins were used } \\
\text { to construct a PPI } \\
\text { network with } 1947 \\
\text { interactions }\end{array}$ & N/A & $\begin{array}{l}\text { Construction of PPI network to } \\
\text { identify regulatory interactions }\end{array}$ & None \\
\hline $\begin{array}{l}\text { Jang et al. } \\
\text { (2014) }\end{array}$ & $\begin{array}{l}\text { Acidogenesis and } \\
\text { solventogenesis. WT, } \\
\text { M5, and M5 + plMP }\end{array}$ & 56 & $\begin{array}{l}\text { All were also identified by } \\
\text { us }\end{array}$ & $\begin{array}{l}\text { Proteins differentially expressed } \\
\text { during acidogenic and } \\
\text { solventogenic phases }\end{array}$ & None \\
\hline
\end{tabular}

Mao et al. (2010) investigated the differential proteome between the WT C. acetobutylicum DSM 1731 (presumably the same strain as the type strain ATCC824) and Rh8 hyper-tolerant butanol mutant and identified 102 differentially expressed protein, among which 86 were identified in the current study (42 and 33 were found to be statistically significant, FDR $5 \%$, under butyrate and butanol stress, respectively) [55].

It was somewhat surprising that a large fraction (23-31\%, by conservative estimation) of the detected proteins in this study displayed opposite differential behavior at the protein versus mRNA level. Disagreements between protein and mRNA levels have been reported in previous studies, all based on microarray data, thus not permitting a detailed interrogation of changes in mRNA composition and structure. These studies included the response of the anaerobe Desulfovibrio vulgaris to low oxygen exposure [63], response in E. coli to different carbon sources [64], adaptation of Streptomyces coelicolor to stationary phase [65], and identification of antibiotic resistance markers in Staphylococcus aureus [66]. In some of these studies, they speculated that such disagreements were due to post-transcriptional regulation $[67,68]$, but no specific mechanisms or explanation were offered except for one, where translational elongation factors were implicated in response to heat shock in a Synechocystis sp. [69]. None of these studies has documented mRNA versus protein disagreements as extensive as those documented here. Significantly, here we provide specific mechanistic explanations based on 5'UTR and RBS strength to explain the mRNA-to-protein expression disagreements for a select set of proteins. Furthermore, we document significant and reproducible changes, across several stress conditions, in the expression of proteins in the translation machinery as well as proteins (e.g., the Rho and S10 (CAC3134) proteins) in transcriptional elongation and termination. These data combined with the identification of differential modification of leaderless transcripts provide support for the hypothesis that different ribosome structures are likely utilized to translate select mRNAs under stress, and also that transcriptional elongation and termination are altered under stress to accommodate the cell's survival program.

Our data provide a rich information basis for more detailed understanding of the complexity of stress response 
but also several target genes/programs that could be engaged for synthetic purposes, that is, for generating strains with superior tolerance to toxic metabolites.

From the fundamental point of view, an area largely unexplored in $\mathrm{Gram}^{+}$organisms like clostridia is the mechanisms that lead to leaderless transcripts and the physiological role of these select leaderless transcripts. The latter, in fact, remains largely unexplored from the evolutionary point of view. What advantage do leaderless transcripts provide for survival under toxic stress? Perhaps the machinery engaged to translate leaderless transcripts is more robust and selected for operation under stress. What is the physiological reason for which the cells have selected the genes that generate leaderless transcripts under physiological versus stress conditions? What is the mechanism for generation of leaderless transcripts in clostridia and other $\mathrm{Gram}^{+}$organisms? What is the role in this context of the Rho protein, of the toxin-antitoxin system(s), and of the specialized translation initiation factors discussed above?

Two other unexplored areas in this and largely all prokaryotes deserve attention and investigation. The first is the apparent employment, strongly suggested by our data, of different components of the translational machinery (and notably of ribosomal proteins) under normal versus stress conditions. This would suggest that different ribosomes are used under different physiological conditions. Is it possible that some ribosomes are unstable under stress (e.g., solvent stress) conditions while others are not? The second area is strongly suggested by our data differential expression of membrane proteins under stress. While logical in many different ways, the molecular mechanisms by which cells make this selection remains virtually unexplored. In this context, the role of the three components of the SRP (the Ffh protein, the SRP non-coding RNA, and the SRP receptor FtsY) and the differential targeting of membrane proteins by SRP under stress deserve detailed investigations from the fundamental but also synthetic point of view. Could we possibly uncover the membrane proteins that result in more rigid membranes to counteract the chaotropic effect of solvents and acids that diminish or destroy the membrane potential and $\Delta \mathrm{pH}$ ? Could we possibly identify the proteins of transporters and related channel proteins that protect the cells from stress through a variety of transport-related mechanisms [4]?

As already stated, our study has identified several genes or programs that could be explored for synthetic applications. Several deserve mentioning here. Engineering cells were based on the SRP system for synthesis of membrane proteins in an exciting possibility. Overexpression and changes in the translational regulation of rubrerythrin should be also explored for enhanced tolerance to acids, oxidative stress, and also likely solvent stress. Understanding the unexplored role of the YacI and $\mathrm{YacH}$ proteins of the $\mathrm{ClpC}$ operon is another exciting possibility. Also, exploring the role of stress protein in a combinatorial fashion remains largely unexplored as a mechanism for enhanced tolerance. While it is well known that most stress proteins work in ensembles and synergistically, very little of that has been explored and only in E. coli $([8,9])$. Is it possible, for example, that the inability of the HSP18 protein to offer enhanced stress tolerance (data not shown), despite the profound upregulation at both the mRNA and protein level, is due to the fact that its partners in action need to also overexpress? Indeed, it is now becoming clear that engineering cells for tolerance is a multicomponent-program goal that requires more sophisticated synthetic biology approaches $[8-10,41,70]$.

An interesting possibility is that these new findings can be mapped on and modeled with the recently reconstructed second-generation genome-scale metabolic model (GSM) using the CoreReg method or variations thereof [71], aiming to dissect the dynamics of cell physiology and gene regulation under stress and its subsequent use for metabolic manipulation for design of robust strains.

\section{Conclusions}

This is the first comprehensive system level study to analyze the stress response in C. acetobutylicum using multi-omic datasets. Significantly, this is the first reported study to systematically engage proteomic and RNAseq data to focus on genes and programs affected by the phenotypic response where post-transcriptional regulation plays a significant role and provide a mechanistic explanation at the system level for such changes. System level understanding of such post-transcriptional regulation can be effectively employed in syntheticbiology and metabolic-engineering strategies for the development of strains with desirable robust traits.

\section{Materials and methods}

\section{Bacterial strains and stress cultures}

Three biological replicates of $C$. acetobutylicum ATCC824 were grown anaerobically in a $\mathrm{pH}$-controlled $(\mathrm{pH}>5)$ batch fermentation in a $4 \mathrm{~L}$ New Brunswick BioFlo 310 bioreactor as described earlier [15, 16]. The cultures were stressed with butyrate (low - $30 \mathrm{mM}$, med $40 \mathrm{mM}$, high - $50 \mathrm{mM}$ ) and butanol (low $-30 \mathrm{mM}$, med $60 \mathrm{mM}$, high - $90 \mathrm{mM}$ ) at mid-exponential growth phase at an OD of 1.0. A non-stressed culture was used as the control. Samples for RNA and protein extractions were obtained at regular intervals of $0,15,30,45,60$, and 
75 min post stress. The proteomic analyses was performed at 15,45 , and $75 \mathrm{~min}$.

\section{Transcriptomic datasets for differential expression analysis}

Transcriptomic analyses were performed using microarrays (GEO datasets GSE48031 and GSE48039) at all six time points [16] and RNAseq [15] (GEO dataset GSE48349) at four time points $(15,30,60$, and $75 \mathrm{~min})$. The data were normalized and analyzed for differential expression using DESeq [72] as described previously [15]. For both transcriptomic techniques, validation was performed using qRT-PCR as reported earlier $[15,16]$.

\section{Strand-specific RNAseq analysis and determination of TSS, UTRs, and operon structures}

Strand-specific RNAseq was performed using libraries from non-stress control, high butanol, and high butyrate stress conditions at two time points, 75 and $270 \mathrm{~min}$ post stress. Following RNA isolation using Qiagen miRNeasy kit and rRNA removal using Ambion MICROBExpress kit, the RNA were also subjected to a Terminator ${ }^{\mathrm{Tx}} 5^{\prime}$-phosphate dependent exonuclease (TEX) treatment for the enrichment of $5^{\prime}$ end of the RNA containing TSS. The libraries were prepared using ScriptSeq V2 (Epicentre, Illumina) and sequenced using paired-end (75 cycles) Illumina HiSeq 2500 at Delaware Biotechnology Institute. Following the trimming [73] of adapters, the data was analyzed using the Rockhopper software [74] for alignment of reads to the reference genome, data normalization, differential expression, TSS prediction, and operon organization. The data has been submitted to NCBI's sequence read archives under the BioProject PRJNA273734 containing 30 BioSamples (SAMN03295242-SAMN03295271).

\section{Protein extraction, digestion, and ITRAQ labeling}

Clostridium acetobutylicum wild-type (WT) cells were cultured under low, medium, and high levels of butanol $(\mathrm{BuOH})$ or butyrate $(\mathrm{BA})$ stress or no stress. For proteomic analysis, cell pellets were resuspended in 100-mL lysis buffer containing $0.01 \%$ SDS and 0.5-M triethylammonium bicarbonate (TEAB) buffer ( $\mathrm{pH} 8.5)$ and sonicated with $1 \%(\mathrm{w} / \mathrm{v})$ calcium carbonate as previously reported [75]. Cell lysates were centrifuged at 20,000 $g$ at $4{ }^{\circ} \mathrm{C}$ for $10 \mathrm{~min}$, and concentration of total protein in the supernatant was determined by Bradford assay (Thermo Fisher Scientific Inc., Rockford, IL, USA). Samples with $100-\mu \mathrm{g}$ protein from each culture condition were reduced, alkylated, digested, and cleaned up as previously reported [75]. The digests were concentrated to $30 \mu \mathrm{L}$ then labeled with iTRAQ 4-plex labels (AB Sciex, Foster City, CA, USA) per manufacturer's instruction according to the labeling scheme listed in Additional file 1: Table S1.

\section{Two-dimensional liquid chromatography (2D-LC) and mass spectrometry (MS) data acquisition}

Labeled samples were combined and separated by high $\mathrm{pH}$ reverse phase LC (RPLC) followed by second dimension RPLC as described [75]. The eluate was introduced to an in-line QTrap 4000 (AB Sciex) through a nanoSpray II source (AB Sciex) using an uncoated fused-silica Pico tip (New Objective, Woburn, MA, USA). MS/MS data were acquired as described [75].

\section{Protein sequence database search and data analysis}

For protein identification and quantification, raw MS/ MS data were submitted to Paragon in ProteinPilot (version $3, \mathrm{AB}$ Sciex) and searched against a local $C A C$ sequence database (a concatenation of NCBI references NC_003030.1 and NC_001988). Search parameters were the same as previously specified [75]. Bias correction and background correction were performed through ProteinPilot. Protein identifications were based on $95 \%$ confidence or above, and only proteins with at least one peptide with $95 \%$ confidence were included in the quantified protein list. For evaluation of the protein identification false discovery rate (FDR), the MS/MS data were submitted to a decoy database and FDR was calculated from the ratio of the number of hits from the decoy database to the number of hits from normal and decoy database (Additional file 2: Proteomic data).

Protein expression levels under $\mathrm{BuOH}$ or $\mathrm{BA}$ stress were compared to the controls under no stress with the same reference using significance analysis of microarrays (SAM analysis [76]) with $\mathrm{MeV}$ v4.8 [77] as reported [75]. The delta values were set for an FDR of $5 \%$ as cutoff.

\section{Additional files}

Additional file 1: Supplemental information. Additional figures and descriptions on integrative proteomic and transcriptomic analyses.

Additional file 2: Proteomic data. Clostridium acetobutylicum

proteomic data for butanol and butyrate stress using iTRAQ labeling.

\section{Abbreviations}

5'UTR: 5' untranslated region; ABE: acetone, butanol, and ethanol; COG: Cluster of Orthologous Groups; HSP: heat shock protein; iTRAQ: isobaric tag for relative and absolute quantitation; RBS: ribosomal binding site; SAM: significant analysis of microarrays; SRP: signal recognition particle; TSS: transcriptional start site.

\section{Competing interests}

The authors declare that they have no competing interests

\section{Authors' contributions}

KPV and SWJ performed the fermentation experiment. LM and SH performed the proteomic analysis. KHL designed the proteomic analyses. MTR prepared the strand-specific libraries and wrote the scripts for the data analyses. KPV performed the microarray experiments and prepared the RNAseq libraries and analyzed the microarray and strand-specific RNAseq data. KPV and ETP prepared the manuscript. ETP conceived the study and the experimental design. ETP and KHL guided the data analysis and interpretation. All authors read and approved the final manuscript. 


\section{Acknowledgements}

The work was supported by the genomic science grant from the Department of Energy, USA (grant \# DE-SC0007092). We thank Qinghua Wang for her help in normalizing the RNAseq data.

\section{Author details}

${ }^{1} 15$ Innovation Way, Delaware Biotechnology Institute, University of Delaware, Newark, DE 19711, USA. ${ }^{2} 150$ Academy Street, Department of Chemical and Biomolecular Engineering, University of Delaware, Newark, DE 19711, USA. ${ }^{3} 15$ Innovation Way, Center for Bioinformatics and Computational Biology, University of Delaware, Newark, DE 19711, USA.

\section{Received: 21 February 2015 Accepted: 30 April 2015}

\section{Published online: 10 June 2015}

\section{References}

1. Papoutsakis ET. Engineering solventogenic clostridia. Curr Opin Biotechnol. 2008;19(5):420-9. doi:10.1016/j.copbio.2008.08.003.

2. Tracy BP, Jones SW, Fast AG, Indurthi DC, Papoutsakis ET. Clostridia: the importance of their exceptional substrate and metabolite diversity for biofuel and biorefinery applications. Curr Opin Biotechnol. 2012;23(3):364-81. doi:10.1016/j.copbio.2011.10.008.

3. Lutke-Eversloh T. Application of new metabolic engineering tools for Clostridium acetobutylicum. Appl Microbiol Biotechnol. 2014;98(13):5823-37. doi:10.1007/s00253-014-5785-5.

4. Nicolaou SA, Gaida SM, Papoutsakis ET. A comparative view of metabolite and substrate stress and tolerance in microbial bioprocessing: from biofuels and chemicals, to biocatalysis and bioremediation. Metab Eng. 2010;12(4):307-31. doi:10.1016/J.Ymben.2010.03.004.

5. Zingaro KA, Nicolaou SA, Papoutsakis ET. Dissecting the assays to assess microbial tolerance to toxic chemicals in bioprocessing. Trends Biotechnol. 2013;31(11):643-53. doi:10.1016/j.tibtech.2013.08.005.

6. Alsaker KV, Paredes C, Papoutsakis ET. Metabolite stress and tolerance in the production of biofuels and chemicals: gene-expression-based systems analysis of butanol, butyrate, and acetate stresses in the anaerobe Clostridium acetobutylicum. Biotechnology and Bioengineering. 2010;105(6):1131-47. doi:10.1002/Bit.22628.

7. Alsaker KV, Spitzer TR, Papoutsakis ET. Transcriptional analysis of spo0A overexpression in Clostridium acetobutylicum and its effect on the cell's response to butanol stress. J Bacteriol. 2004;186(7):1959-71. doi:10.1128/ Jb.186.7.1959-1971.2004.

8. Zingaro KA, Papoutsakis ET. Toward a semisynthetic stress response system to engineer microbial solvent tolerance. Mbio. 2012;3((5):e00308-12. doi:10.1128/mBio.00308-12.

9. Zingaro KA, Papoutsakis ET. GroESL overexpression imparts Escherichia coli tolerance to i-, n-, and 2-butanol, 1,2,4-butanetriol and ethanol with complex and unpredictable patterns. Metab Eng. 2013;15:196-205. doi:10.1016/j.ymben.2012.07.009.

10. Zingaro KA, Nicolaou SA, Yuan YB, Papoutsakis ET. Exploring the heterologous genomic space for building, stepwise, complex, multicomponent tolerance to toxic chemicals. ACS Synth Biol. 2014:3(7):476-86. doi:10.1021/sb400156v.

11. Tomas CA, Welker NE, Papoutsakis ET. Overexpression of groESL in Clostridium acetobutylicum results in increased solvent production and tolerance, prolonged metabolism, and changes in the cell's transcriptional program. Appl Environ Microbiol. 2003;69(8):4951-65. doi:10.1128/ Aem.69.8.4951-4965.2003.

12. Alsaker KV, Papoutsakis ET. Transcriptional program of early sporulation and stationary-phase events in Clostridium acetobutylicum. J Bacterol. 2005;187(20):7103-18. doi:10.1128/Jb.187.20.7103-7118.2005.

13. Jones SW, Paredes CJ, Tracy B, Cheng N, Sillers R, Senger RS, et al. The transcriptional program underlying the physiology of clostridial sporulation. Genome Biol. 2008;9(7):R114. doi:10.1186/gb-2008-9-7-r114.

14. Tomas CA, Beamish J, Papoutsakis ET. Transcriptional analysis of butanol stress and tolerance in Clostridium acetobutylicum. J Bacteriol. 2004;186(7):2006-18.

15. Venkataramanan KP, Jones SW, McCormick KP, Kunjeti SG, Ralston MT Meyers BC. The clostridium small RNome that responds to stress: the paradigm and importance of toxic metabolite stress in C. acetobutylicum. BMC Genomics. 2013;14:849. doi:10.1186/1471-2164-14-849.
16. Wang $\mathrm{Q}$, Venkataramanan $\mathrm{KP}$, Huang $\mathrm{H}$, Papoutsakis ET, Wu CH. Transcription factors and genetic circuits orchestrating the complex, multilayered response of Clostridium acetobutylicum to butanol and butyrate stress. BMC Syst Biol. 2013;7(1):120. doi:10.1186/1752-0509-7-120

17. Janssen H, Grimmler C, Ehrenreich A, Bahl H, Fischer RJ. A transcriptional study of acidogenic chemostat cells of Clostridium acetobutylicum - Solvent stress caused by a transient n-butanol pulse. J Biotechnol. 2012;161(3):354-65. doi:10.1016/j.jbiotec.2012.03.027.

18. Schwarz KM, Kuit W, Grimmler C, Ehrenreich A, Kengen SWM. A transcriptional study of acidogenic chemostat cells of Clostridium acetobutylicum — cellular behavior in adaptation to n-butanol. J Biotechnol. 2012;161(3):366-77. doi:10.1016/j.jbiotec.2012.03.018.

19. Zhang W, Li F, Nie L. Integrating multiple 'omics' analysis for microbial biology: application and methodologies. Microbiology. 2010;156(Pt 2):287-301. doi:10.1099/mic.0.034793-0.

20. Venkataramanan KP, Kurniawan Y, Boatman JJ, Haynes $\mathrm{CH}$, Taconi KA, Martin $L$, et al. Homeoviscous response of Clostridium pasteurianum to butanol toxicity during glycerol fermentation. J Biotechnol. 2014;179:8-14. doi:10.1016/j.jbiotec.2014.03.017.

21. Wang FQ, Kashket S, Kashket ER. Maintenance of Delta $\mathrm{pH}$ by a butanol-tolerant mutant of Clostridium beijerinckii. Microbiol-Sgm. 2005;151:607-13. doi:10.1099/mic.0.27587-0.

22. Papoutsakis ET, Bussineau CM, Chu IM, Diwan AR, Huesemann M. Transport of substrates and metabolites and their effect on cell-metabolism (in butyric-acid and methylotrophic fermentations). Ann N Y Acad Sci. 1987;506:24-50. doi:10.1111/j.1749-6632.1987.tb23808.x.

23. Boudvillain M, Figueroa-Bossi N, Bossi L. Terminator still moving forward: expanding roles for Rho factor. Curr Opin Microbiol. 2013;16(2):118-24. doi:10.1016/j.mib.2012.12.003.

24. Roberts JW, Shankar S, Filter JJ. RNA polymerase elongation factors. Annual Review of Microbiology Annual Review of Microbiology. 2008;62:211-33.

25. Wang C, Gong BS, Bushel PR, Thierry-Mieg J, Thierry-Mieg D, Xu JS. The concordance between RNA-seq and microarray data depends on chemical treatment and transcript abundance. Nat Biotechnol. 2014;32(9):926-32. doi:10.1038/Nbt.3001.

26. Wietzke $\mathrm{M}$, Bahl $\mathrm{H}$. The redox-sensing protein Rex, a transcriptional regulator of solventogenesis in Clostridium acetobutylicum. Appl Microbiol Biotechnol. 2012;96(3):749-61. doi:10.1007/s00253-012-4112-2.

27. Moll I, Engelberg-Kulka H. Selective translation during stress in Escherichia coli. Trends Biochem Sci. 2012;37(11):493-8. doi:10.1016/j.tibs.2012.07.007.

28. Vesper O, Amitai S, Belitsky M, Byrgazov K, Kaberdina AC, Engelberg-Kulka H, et al. Selective translation of leaderless mRNAs by specialized ribosomes generated by MazF in Escherichia coli. Cell. 2011;147(1):147-57. doi:10.1016/ j.cell.2011.07.047.

29. Paredes CJ, Rigoutsos I, Papoutsakis ET. Transcriptional organization of the Clostridium acetobutylicum genome. Nucleic Acids Res. 2004;32(6):1973-81. doi:10.1093/nar/gkh509.

30. Cortes T, Schubert OT, Rose G, Arnvig KB, Comas I, Aebersold R, et al. Genome-wide mapping of transcriptional start sites defines an extensive leaderless transcriptome in Mycobacterium tuberculosis. Cell Rep. 2013;5(4):1121-31. doi:10.1016/j.celrep.2013.10.031.

31. O'Donnell SM, Janssen GR. The initiation codon affects ribosome binding and translational efficiency in Escherichia coli of $\mathrm{cl}$ mRNA with or without the 5' untranslated leader. J Bacteriol. 2001;183(4):1277-83. doi:10.1128/ Jb.183.4.1277-1283.2001.

32. Schluter JP, Reinkensmeier J, Barnett MJ, Lang C, Krol E, Giegerich R, et al. Global mapping of transcription start sites and promoter motifs in the symbiotic alpha-proteobacterium Sinorhizobium meliloti 1021. BMC Genomics. 2013;14:156. doi:10.1186/1471-2164-14-156.

33. Zheng $X B, H u G Q$, She $Z \mathrm{~S}, \mathrm{Zhu} H \mathrm{HQ}$. Leaderless genes in bacteria: clue to the evolution of translation initiation mechanisms in prokaryotes. BMC Genomics. 2011;12:Artn 361. doi:10.1186/1471-2164-12-361.

34. Moll I, Grill S, Gualerzi CO, Blasi U. Leaderless mRNAs in bacteria: surprises in ribosomal recruitment and translational control. Mol Microbiol. 2002;43(1):239-46.

35. Yang SH, Pan CL, Tschaplinski TJ, Hurst GB, Engle NL, Zhou W. Systems biology analysis of Zymomonas mobilis ZM4 ethanol stress responses. PLoS One. 2013;8(7):e68886. doi:10.1371/journal.pone.0068886.

36. Jüschke $C$, Dohnal I, Pichler P, Harzer $H$, Swart R, Ammerer $G$, et al. Transcriptome and proteome quantification of a tumor model provides novel insights into post-transcriptional gene regulation. Genome Biol. 2013;14(11):r133 
37. Lackner DH, Schmidt MW, Wu S, Wolf DA, Bahler J. Regulation of transcriptome, translation, and proteome in response to environmental stress in fission yeast. Genome Biol. 2012;13(4):R25. doi:10.1186/gb-2012-13-4-r25.

38. Vellanoweth $\mathrm{RL}$, Rabinowitz JC. The influence of ribosome-binding-site elements on translational efficiency in Bacillus subtilis and Escherichia coli in vivo. Mol Microbiol. 1992;6(9):1105-14.

39. Gustafsson C, Govindarajan S, Minshull J. Codon bias and heterologous protein expression. Trends Biotechnol. 2004;22(7):346-53. doi:10.1016/ j.tibtech.2004.04.006.

40. Borujeni AE, Channarasappa AS, Salis HM. Translation rate is controlled by coupled trade-offs between site accessibility, selective RNA unfolding and sliding at upstream standby sites. Nucleic Acids Res. 2014;42(4):2646-59. doi:10.1093/Nar/Gkt1139.

41. Gaida SM, Al-Hinai MA, Indurthi DC, Nicolaou SA, Papoutsakis ET. Synthetic tolerance: three noncoding small RNAs, DsrA, ArcZ and RprA, acting supra-additively against acid stress. Nucleic Acids Res. 2013;41(18):8726-37. doi:10.1093/nar/gkt651.

42. Sharma CM, Darfeuille F, Plantinga TH, Vogel J. Small RNA regulates multiple $A B C$ transporter mRNAs by targeting C/A-rich elements inside and upstream of ribosome-binding sites. Genes Dev. 2007;21(21):2804-17. doi:10.1101/Gad.447207.

43. Kroger C, Dillon SC, Cameron AD, Papenfort K, Sivasankaran SK, Hokamp K, et al. The transcriptional landscape and small RNAs of Salmonella enterica serovar Typhimurium. Proc Natl Acad Sci U S A. 2012;109(20):E1277-86. doi:10.1073/pnas.1201061109.

44. Hyatt D, Chen GL, Locascio PF, Land ML, Larimer FW, Hauser L. Prodigal: prokaryotic gene recognition and translation initiation site identification. BMC bioinformatics. 2010;11:119. doi:10.1186/1471-2105-11-119.

45. Hillmann F, Fischer R-J, Bahl H. The rubrerythrin-like protein Hsp21 of Clostridium acetobutylicum is a general stress protein. Arch Microbiol. 2006;185(4):270-6.

46. May A, Hillmann F, Riebe O, Fischer RJ, Bahl H. A rubrerythrin-like oxidative stress protein of Clostridium acetobutylicum is encoded by a duplicated gene and identical to the heat shock protein Hsp21. FEMS Microbiol Lett. 2004;238(1):249-54.

47. Jain S, Graham C, Graham RL, McMullan G, Ternan NG. Quantitative proteomic analysis of the heat stress response in Clostridium difficile strain 630. J Proteome Res. 2011;10(9):3880-90. doi:10.1021/Pr200327t.

48. Jean D, Briolat V, Reysset G. Oxidative stress response in Clostridium perfringens. Microbiol-Sgm. 2004;150:1649-59. doi:10.1099/mic.0.27017-0.

49. Hillmann F, Riebe O, Fischer RJ, Mot A, Caranto JD, Kurtz Jr DM, et al. Reductive dioxygen scavenging by flavo-diiron proteins of Clostridium acetobutylicum. FEBS Lett. 2009;583(1):241-5. doi:10.1016/j.febslet.2008.12.004.

50. Sledjeski DD, Gupta A, Gottesman S. The small RNA, DsrA, is essential for the low temperature expression of RpoS during exponential growth in Escherichia coli. Embo Journal. 1996;15(15):3993-4000.

51. Gottesman S, McCullen C, Guillier M, Vanderpool C, Majdalani N, Benhammou J et al., editors. Small RNA regulators and the bacterial response to stress. In Cold Spring Harbor Symposia on Quantitative Biology, vol. 71, pp. 1-11. Cold Spring Harbor Laboratory Press, 2006.

52. Hatzimanikatis $\mathrm{V}$, Lee $\mathrm{KH}$. Dynamical analysis of gene networks requires both mRNA and protein expression information. Metab Eng. 1999;1(4):275-81.

53. Honicke D, Janssen H, Grimmler C, Ehrenreich A, Lutke-Eversloh T. Global transcriptional changes of Clostridium acetobutylicum cultures with increased butanol:acetone ratios. N Biotechnol. 2012;29(4):485-93. doi:10.1016/ j.nbt.2012.01.001.

54. Janssen H, Doring C, Ehrenreich A, Voigt B, Hecker M, Bahl H. A proteomic and transcriptional view of acidogenic and solventogenic steady-state cells of Clostridium acetobutylicum in a chemostat culture. Appl Microbiol Biotechnol. 2010;87(6):2209-26. doi:10.1007/s00253-010-2741-x.

55. Mao SM, Luo YAM, Zhang TR, Li JS, Bao GAH, Zhu Y. Proteome reference map and comparative proteomic analysis between a wild type Clostridium acetobutylicum DSM 1731 and its mutant with enhanced butanol tolerance and butanol yield. J Proteome Res. 2010;9(6):3046-61. doi:10.1021/Pr9012078

56. Schaffer S, Isci N, Zickner B, Durre P. Changes in protein synthesis and identification of proteins specifically induced during solventogenesis in Clostridium acetobutylicum. Electrophoresis. 2002;23(1):110-21. doi:10.1002/ 1522-2683(200201)23:1<110::AID-ELPS110>3.0.CO;2-G.
57. Sullivan L, Bennett GN. Proteome analysis and comparison of Clostridium acetobutylicum ATCC 824 and Spo0A strain variants. J Ind Microbiol Biotechnol. 2006;33(4):298-308. doi:10.1007/s10295-005-0050-7.

58. Bai $\mathrm{X}, \mathrm{Ji} \mathrm{ZH}$. Phosphoproteomic investigation of a solvent producing bacterium Clostridium acetobutylicum. Appl Microbiol Biotechnol. 2012;9(1):201-11. doi:10.1007/s00253-012-4156-3.

59. Mao S, Luo Y, Bao G, Zhang Y, Li Y, Ma Y. Comparative analysis on the membrane proteome of Clostridium acetobutylicum wild type strain and its butanol-tolerant mutant. Molecular BioSystems. 2011;7(5):1660-77.

60. Sivagnanam K, Raghavan VGS, Shah M, Hettich RL, Verberkmoes NC, Lefsrud MG. Comparative shotgun proteomic analysis of Clostridium acetobutylicum from butanol fermentation using glucose and xylose. Proteome Sci. 2011;9(1):66. doi:10.1186/1477-5956-9-66.

61. Sivagnanam K, Raghavan VGS, Shah M, Hettich RL, Verberkmoes NC. Lefsrud. MG Characterization of Clostridium Acetobutylicum Protein Interaction Network from Butanol Fermentation J Anal Bioanal Tech. 2012;\$3:002. doi:10.4172/2155-9872.S3-002

62. Jang YS, Han MJ, Lee J, Im JA, Lee YH, Papoutsakis ET, et al. Proteomic analyses of the phase transition from acidogenesis to solventogenesis using solventogenic and non-solventogenic Clostridium acetobutylicum strains. Appl Microbiol Biotechnol. 2014;98(11):5105-15.

63. Mukhopadhyay A, Redding AM, Joachimiak MP, Arkin AP, Borglin SE, Dehal PS, et al. Cell-wide responses to low-oxygen exposure in Desulfovibrio vulgaris Hildenborough. J Bacteriol. 2007;189(16):5996-6010. doi:10.1128/JB.00368-07.

64. Corbin RW, Paliy O, Yang F, Shabanowitz J, Platt M, Lyons Jr CE, et al. Toward a protein profile of Escherichia coli: comparison to its transcription profile. Proc Natl Acad Sci U S A. 2003;100(16):9232-7. doi:10.1073/ pnas. 1533294100.

65. Jayapal KP, Philp RJ, Kok YJ, Yap MG, Sherman DH, Griffin TJ, et al. Uncovering genes with divergent mRNA-protein dynamics in Streptomyces coelicolor. PLoS One. 2008;3(5), e2097. doi:10.1371/journal.pone.0002097.

66. Scherl A, Francois P, Charbonnier Y, Deshusses JM, Koessler T, Huyghe A, et al. Exploring glycopeptide-resistance in Staphylococcus aureus: a combined proteomics and transcriptomics approach for the identification of resistance-related markers. BMC Genomics. 2006;7:296. doi:10.1186/1471-2164-7-296.

67. Budde I, Steil L, Scharf C, Volker U, Bremer E. Adaptation of Bacillus subtilis to growth at low temperature: a combined transcriptomic and proteomic appraisal. Microbiol-Sgm. 2006;152:831-53. doi:10.1099/mic.0.28530-0.

68. Schmid AK, Reiss DJ, Kaur A, Pan M, King N, Van PT, et al. The anatomy of microbial cell state transitions in response to oxygen. Genome Res. 2007;17(10):1399-413. doi:10.1101/gr.6728007.

69. Suzuki I, Simon WJ, Slabas AR. The heat shock response of Synechocystis sp. PCC 6803 analysed by transcriptomics and proteomics. J Exp Bot. 2006;57(7):1573-8. doi:10.1093/jxb/erj148.

70. Nicolaou SA, Gaida SM, Papoutsakis ET. Exploring the combinatorial genomic space in Escherichia coli for ethanol tolerance. Biotechnol J. 2012;7(11):1337-45. doi:10.1002/biot.201200227.

71. Dash S, Mueller TJ, Venkataramanan KP, Papoutsakis ET, Maranas CD. Capturing the response of Clostridium acetobutylicum to chemical stressors using a regulated genome-scale metabolic model. Biotechnology for biofuels. 2014;7(1):144.

72. Anders S, Huber W. Differential expression analysis for sequence count data. Genome Biol. 2010;11(10):Artn R106. doi:10.1186/Gb-2010-11-10-R106.

73. Bolger AM, Lohse M, Usadel B. Trimmomatic: a flexible trimmer for Illumina sequence data. Bioinformatics. 2014;30(15):2114-20. doi:10.1093/ bioinformatics/btu170.

74. McClure R, Balasubramanian D, Sun Y, Bobrovskyy M, Sumby P, Genco CA, et al. Computational analysis of bacterial RNA-Seq data. Nucleic Acids Res. 2013;41(14), e140. doi:10.1093/nar/gkt444.

75. Hou S, Jones SW, Choe LH, Papoutsakis ET, Lee KH. Workflow for quantitative proteomic analysis of Clostridium acetobutylicum ATCC 824 using iTRAQ tags. Methods. 2013;61(3):269-76. doi:10.1016/ j.ymeth.2013.03.013.

76. Tusher VG, Tibshirani R, Chu G. Significance analysis of microarrays applied to the ionizing radiation response. Proc Natl Acad Sci U S A. 2001;98(9):5116-21. doi:10.1073/pnas.091062498.

77. Saeed Al, Sharov V, White J, Li J, Liang W, Bhagabati N, et al. TM4: a free, open-source system for microarray data management and analysis. Biotechniques. 2003;34(2):374-8. 